\title{
Effect of high frequency vibrations on PV silicon purification
}

\author{
Samia Bouarab ${ }^{1,2}$, Faiza Mokhtari ${ }^{1}$, Slim Kaddeche $^{3}$, Daniel Henry ${ }^{4}$, Valéry Botton ${ }^{4,5}$, \\ Abdessamed Medelfef ${ }^{1,4}$ \\ ${ }^{1}$ Laboratoire de Thermodynamique et Systèmes Energétiques, Faculté de Physique, Université des Sciences et de \\ la Technologie Houari Boumediène, BP 32, 16111 Bab Ezzouar, Alger, Algeria \\ ${ }^{2}$ Université M'Hammed Bougara, Boumerdes, Algeria \\ ${ }^{3}$ Laboratoire de Recherche Matériaux, Mesures et Applications LR-11-ES-25, Institut National des Sciences \\ Appliquées et de Technologie, BP 676, 1080 Tunis Cedex, Tunisia \\ ${ }^{4}$ Laboratoire de Mécanique des Fluides et d'Acoustique, CNRS, Université de Lyon, \\ Ecole Centrale de Lyon/ Université Lyon 1/INSA Lyon, ECL, 36 Avenue Guy de Collongue, 69134 Ecully \\ Cedex, France \\ ${ }^{5}$ INSA Euro-Méditerranée, Université Euro-Méditerranéenne de Fès, Route de Meknès, BP51, Fez, Morocco
}

\begin{abstract}
Silicon purification for photovoltaic applications is a crucial challenge to improve the energy production performance of solar panels. In the present work, we focus on the metallurgical grade silicon purification process through a directional solidification technique, namely the horizontal Bridgman technique. By means of numerical simulations, it is shown that high frequency vibrations applied to the crucible can be used to enhance the convective level in the molten silicon (thermal-vibrational effect) and improve the purification of the final silicon ingot. The direction of the vibration, however, has to be carefully chosen, as it strongly influences the flow intensity and structure in the melt and can lead to multi-rolls and even reverse flows, with a direct effect on the induced purification.
\end{abstract}

Keywords: A1. Directional solidification, A1. Convection, A1. Segregation, A1. Purification, A1. Vibrations

\section{Introduction}

The principal material used in Photovoltaic (PV) cells is silicon and especially multi-crystalline silicon. In order to reduce the production costs of photovoltaic cells, and to achieve large ingots, the metallurgical grade silicon (MG-Si) is used, which has then to be transformed into solar grade silicon (SG-Si) by different techniques. To reach a high level of solar energy conversion, the solar grade silicon used to achieve PV solar cells must satisfy to very high purity criteria $(99.9999 \%)$, whereas the metallurgical grade silicon purity is generally around $98 \%$. Two types of impurities can exist in silicon, dopants, intentionally incorporated to achieve semiconductor crystals with specific electronic properties, and undesirable impurities such as phosphorus, iron, and aluminum, which are naturally present in the metallurgical grade silicon. Reducing the level of impurities (contaminants such as phosphorus present in relatively significant concentrations) is highly requested to improve the solar energy conversion efficiency of PV silicon cells. A compromise, however, has to be found between the choice of a relevant technique to purify metallurgical grade silicon and its cost of implementation, and this represents a real industrial challenge.

In order to obtain silicon with better levels of purity, several purification techniques such as volatile vaporization, solvent refining, and slag treatment have been implemented [1-4]. However, the implementation cost of these techniques is somewhat high due to the high temperatures required and most of them can be at the origin of silicon loss during processing. Si purification can also be obtained by directional solidification steps, due to the fact that at solid-liquid phase equilibrium, the concentration of impurities in the solid phase is less than in 
the liquid phase. Practically, the impurities will be more or less difficult to remove depending on their thermophysical properties and especially their segregation coefficient, and those with a low segregation coefficient as aluminum will be the most easily removed by directional solidification. In order to optimize the purification of metallurgical grade silicon by directional solidification, a number of active control techniques are used. Among these techniques, we can mention mechanical stirring process [5-6], the use of different types of magnetic fields [7-8], the rotation of the crucible as in the Accelerated Crucible Rotation Technique or vibrational techniques as the Axial Vibration Control using vibrations of the growing crystal or oscillation of a submerged body (see references for these techniques in [9]), and the acoustic streaming (steady flow generated by progressive acoustic waves) [10]. The main objective of these control techniques is to enhance the level of mixing in the melt, in the vicinity of the growth interface, in order to achieve silicon ingots containing less impurities. Impurities segregation during directional solidification processes has also been investigated in different papers taking into account the rejection of solute at the solid-liquid front and its transport by diffusion and natural convection [11-17].

In the present work, we propose to use high-frequency vibrations of the crucible to control the flow and therefore the heat and mass transfer in the melt, with the objective to reduce the rate of impurities in silicon ingots grown by the horizontal Bridgman technique. As indicated by Gershuni and Lyubimov [18], in a closed cavity submitted to vibrations in the absence of temperature non-uniformities, the fluid moves together with the cavity as a solid body. However, as discussed in detail in [18], in presence of a temperature gradient, a relative flow will appear. In fact, the time average of the cross product between the non-uniform temperature field and the imposed acceleration due to the vibrations is non zero, resulting in an additional term in the Navier-Stokes equation for the average flow. The excitation frequencies must be not too high to avoid cavitation phenomena. To give order of magnitudes, the frequency range to be considered extends from a few hundred $\mathrm{Hz}$ up to a few $\mathrm{MHz}$, with oscillation amplitudes below $\mathrm{mm}$ down to $\mu \mathrm{m}$. Various theoretical and experimental studies of thermal-vibrational convection (such convection occurring in heated fluids submitted to high-frequency vibrations), in microgravity as well as in ground conditions and for various configurations, are available in the literature [18-25]. In these references, it has been proved that the vibrations have an impact on both the flow pattern and its convective level. Depending on the direction, the amplitude and the frequency of the vibrations imposed to the crucible, a slowing down or an enhancement of the flow has been highlighted. The vibration conditions that induce an amplification of the convective level are expected to be able to promote mixing in the melt and thus could be used to improve silicon purification. Despite the large number of theoretical and experimental works about directional solidification and about thermal-vibrational convection, we did not find any study on the use of thermal-vibrational convection to improve silicon purification by directional solidification.

Our numerical study is thus devoted to the effect of high frequency vibrations of the crucible on purification of metallurgical grade silicon for photovoltaic applications. The results are obtained in the horizontal Bridgman situation, for an enclosure with an initial aspect ratio $A=$ 4 filled with molten metallurgical grade silicon $(\mathrm{Pr}=0.0113)$. The influence of the vibrations on the impurities concentration in the melt and in the silicon ingot during the solidification of half the initial melt is characterized for different intensities and directions of vibrations.

\section{Mathematical formulation}

The horizontal Bridgman setup considered in this work is depicted in Fig. 1. The crucible, filled with molten silicon, is a rectangular cavity with an aspect ratio $A=L / H=4$, where $L$ is its length and $H$ is its height. It is pulled at a constant speed $V_{f}$ inside a constant gradient furnace 
delivering a uniform horizontal temperature gradient $\overline{\nabla T}$ (Ben Hadid and Roux [26], Kaddeche et al. [14-15]), $\overline{\nabla T}$ is the absolute gradient, corresponding then to a positive value. The molten silicon will then progressively solidify, giving a liquid part with a decreasing length $L_{l}$. High frequency vibrations are applied to the crucible. These vibrations have a direction given by the unit vector $\boldsymbol{n}$ (which makes an angle $\alpha_{V}$ with the horizontal), an amplitude $b$ and an angular frequency $\Omega$. The angle $\alpha_{V}$ has a period of $\pi$ or $180^{\circ}$. The vibrations induce an additional vibrational component to the gravitational acceleration $\boldsymbol{g}_{\mathbf{0}}$, giving a resulting acceleration $\boldsymbol{g}=$ $\boldsymbol{g}_{\mathbf{0}}+b \Omega^{2} \sin (\Omega t) \boldsymbol{n}[18]$.

The impurities concentration in the metallurgical silicon remains small, so that the density variations in the molten silicon can be considered as only due to the temperature variations and independent of the solutal effects (dilute alloy assumption). The melt is assumed to be incompressible and Newtonian, and to satisfy the Boussinesq approximation, and the flow is assumed to be laminar. As the pulling rate $V_{f}$ is considered to be weak (classically in the 1-10 $\mu \mathrm{m} / \mathrm{s}$ range), the thermodynamic equilibrium is satisfied during the whole solidification process with a constant segregation coefficient $k$. As the melt is also a good thermal conductor, the moderate flows considered in the study will not strongly perturb the temperature field and the heat transfer can be considered to proceed mainly by diffusion with almost vertical straight isotherms (see Fig. 4(a) as an example). As a consequence, the crystal-melt interface can be considered as planar and moving at a constant velocity $-V_{f}$ deduced from the pulling rate.

Under these assumptions and in presence of vibrations, the governing time-dependent equations in the melt, expressed in terms of dimensionless temperature $T$, impurity concentration $C$, and stream functions $\psi$ and $F$ associated with the average velocity $\boldsymbol{V}$ and the pulsation velocity $\boldsymbol{W}$ induced by the vibrations, respectively $[18,25]$, can be written as follows:

$$
\begin{aligned}
& \frac{\partial \nabla^{2} \psi}{\partial t}+\left(\frac{\partial \psi}{\partial x} \frac{\partial \nabla^{2} \psi}{\partial z}+\left(-\frac{\partial \psi}{\partial z}-\frac{x}{S} v_{f}\right) \frac{\partial \nabla^{2} \psi}{\partial x}\right)=\nabla^{4} \psi+\mathrm{Gr} \frac{\partial T}{\partial x} \\
& +\mathrm{Gr}_{\mathrm{v}}\left[\left(\frac{\partial^{2} F}{\partial x^{2}} \frac{\partial T}{\partial z}-\frac{\partial^{2} F}{\partial x \partial z} \frac{\partial T}{\partial x}\right) \sin \alpha_{V}-\left(\frac{\partial^{2} F}{\partial x \partial z} \frac{\partial T}{\partial z}-\frac{\partial^{2} F}{\partial z^{2}} \frac{\partial T}{\partial x}\right) \cos \alpha_{V}\right] \\
& \frac{\partial T}{\partial t}+\left(\frac{\partial \psi}{\partial x} \frac{\partial T}{\partial z}+\left(-\frac{\partial \psi}{\partial z}-\frac{x}{S} v_{f}\right) \frac{\partial T}{\partial x}\right)=\frac{1}{\operatorname{Pr}} \nabla^{2} T \\
& \frac{\partial C}{\partial t}+\left(\frac{\partial \psi}{\partial x} \frac{\partial C}{\partial z}+\left(-\frac{\partial \psi}{\partial z}-\frac{x}{S} v_{f}\right) \frac{\partial C}{\partial x}\right)=\frac{1}{\operatorname{Sc}} \nabla^{2} C \\
& \nabla^{2} F=\frac{\partial T}{\partial x} \sin \alpha_{V}-\frac{\partial T}{\partial z} \cos \alpha_{V}
\end{aligned}
$$

The stream functions $\psi$ and $F$ are defined as,

$$
V_{x}=-\frac{\partial \psi}{\partial z}, \quad V_{z}=\frac{\partial \psi}{\partial x}, \quad W_{x}=-\frac{\partial F}{\partial z}, \quad W_{z}=\frac{\partial F}{\partial x}
$$

where $V_{x}$ and $V_{z}$ are the components of the average velocity $\boldsymbol{V}$, and $W_{x}$ and $W_{z}$ are the components of the pulsation velocity $\boldsymbol{W}$.

In the dimensionless equations (1)-(4), length has been scaled by the crucible height $H$, time by $H^{2} / v$, velocity by $v / H$, concentration by $\overline{C_{0}}$ (the initial impurity concentration in the melt), and temperature and pulsation velocity by $\overline{\nabla T} H . v$ is the kinematic viscosity of the molten silicon. 
$S(t)$ is the dimensionless length (or aspect ratio) of the melt $\left(S=L_{l} / H\right)$, which varies during the growth process from $A$ at the beginning of the solidification to $A / 2$ when half of the total melt is solidified, and $v_{f}=-V_{f} H / v$ is the dimensionless solid-liquid interface velocity. As the interface moves along the $x$ direction from the right to the left of the crucible (decreasing $x$ ), $v_{f}$ will be negative. The dimensionless temperature is defined as $T=\left(\bar{T}-T_{m}\right) /(\overline{\nabla T} H)$, where $\bar{T}$ is the dimensional temperature and $T_{m}$ is the melting temperature of silicon, so that $T$ is 0 at the solid-liquid interface at $x=S$ and $S$ at the left crucible wall at $x=0$. The dimensionless concentration is defined as $C=\bar{C} / \overline{C_{0}}$, where $\bar{C}$ is the dimensional concentration.

The dimensionless parameters involved in these equations, namely the Prandtl number Pr, the Schmidt number Sc, the Grashof number Gr and the vibrational Grashof number $\mathrm{Gr}_{\mathrm{v}}$ are given by:

$$
\operatorname{Pr}=\frac{v}{\chi}, \quad \mathrm{Sc}=\frac{v}{D}, \quad \mathrm{Gr}=\frac{g_{0} \beta \overline{\nabla T} H^{4}}{v^{2}} \quad \text { and } \quad \mathrm{Gr}_{\mathrm{v}}=\frac{(b \Omega \beta \overline{\nabla T})^{2} H^{4}}{2 v^{2}}
$$

where $\beta$ and $\chi$ are the thermal expansion coefficient and the thermal diffusivity of the molten silicon, respectively, and $D$ is the impurity diffusivity.

The concentration in the crystal $C_{s}$ is deduced at each time step from the liquid concentration $C$ at the crystal-melt interface through the equilibrium relationship $C_{s}=k C$. The segregation coefficient $k$ is considered as a constant depending on the considered impurity.

The boundary conditions for the velocity correspond to no-slip conditions for the average velocity $\boldsymbol{V}$ and a nonpermeability condition for the pulsation velocity $\boldsymbol{W}[18,25]$. Concerning the temperature, the boundary conditions are imposed by the constant gradient furnace: the vertical walls have uniform temperatures, $\bar{T}=T_{m}$ at the interface and $\bar{T}=T_{m}+\overline{\nabla T} L_{l}$ at the left crucible wall, and the horizontal walls have a linear temperature variation between these two limit temperatures. Finally, concerning concentration, a balance between the impurity reject and its diffusion in the liquid is applied at the solid-liquid interface, whereas a zero mass-flux is considered at the other walls.

With these assumptions, the boundary conditions can be written as follows:

At the vertical rigid boundary $x=0$ :

$$
\psi=\frac{\partial \psi}{\partial x}=0, \quad F=0, \quad T=S \text { and } \frac{\partial C}{\partial x}=0
$$

At the crystal-melt interface $x=S$ :

$$
\psi=\frac{\partial \psi}{\partial x}=0, \quad F=0, T=0 \text { and } \frac{\partial C}{\partial x}=(1-k) \operatorname{Sc} v_{f} C
$$

At the bottom $z=0$ :

$$
\psi=\frac{\partial \psi}{\partial z}=0, \quad F=0, T=S-x \text { and } \frac{\partial C}{\partial z}=0
$$

At the upper boundary $z=1$ :

$$
\psi=\frac{\partial \psi}{\partial z}=0, \quad F=0, \quad T=S-x \text { and } \frac{\partial C}{\partial z}=0
$$

Finally, we have to note that the thermal gradient chosen here in accordance with previous simulations of solidification [14-15] (Fig. 1) is opposite to that generally used in thermalvibrational convection studies [24-25]. As a consequence, the optimal vibration direction giving the stronger flow will not be obtained as usually for $45^{\circ}$, but for $\alpha_{V}=135^{\circ}$. In order to keep the angle $45^{\circ}$ for this optimal direction, we will then define $\alpha=180^{\circ}-\alpha_{V}$. This angle $\alpha$ will be called the vibration angle in the following. 


\section{Numerical method}

The governing equations (1)-(4) subjected to the relevant boundary conditions (5)-(8) are solved with the highly accurate spectral collocation method on Gauss-Lobatto-Chebyshev points, as described in the work of Bouarab et al. [25]. The time stepping for these unsteady calculations is based on Euler's method, which, due to the small time steps used for an accurate treatment of the interface conditions, is shown to give similar results as the Crank-Nicolson method. For validation and accuracy tests of the vibration effect on convection, we refer to the recent results of Bouarab et al. [25] and also to the earlier work of Lappa [24]. Concerning the segregation effect in both liquid and solid phases, our results can be compared with those of Kaddeche et al. [14] corresponding to Ge-Ga or GaAs-In alloys and obtained for $\operatorname{Pr}=0.015, v_{f}=-0.2$, $k=0.087$, and different values of the Grashof and Schmidt numbers in the case without vibrations. For both hydrodynamics and segregation calculations, our results are found to be in excellent agreement with these previous works.

\section{Results and discussion}

\subsection{Effect of vibrations on the convective flows}

Having in mind that a sufficiently high convective level is a favorable condition to get grown crystals containing less impurities [14,15], a purely hydrodynamic study without phase change is first conducted to find out the characteristics of the vibrations allowing to obtain the highest convective level in the liquid phase. Numerical computations are first performed for $A=4$, $\operatorname{Pr}=0.0113$, different values of the Grashof and vibrational Grashof numbers $\mathrm{Gr}$ and $\mathrm{Gr}_{\mathrm{v}}$, and all the possible orientations of the vibrations, for $\alpha$ between 0 and $180^{\circ}$. The results are shown in Fig. 2, where, for the different cases, the maximum velocity in the cavity is plotted as a function of $\alpha$. It can be clearly seen that the maximum flow intensity varies with the vibration angle $\alpha$ and that it reaches a maximum value for vibrations corresponding to $\alpha$ close to $45^{\circ}$. It confirms the results obtained by Bouarab et al. [25] in a different situation with thermally insulated top and bottom walls.

The enhancement of the convective level, however, has mainly to be effective in the vicinity of the growth interface where the reject of impurities from the solid to the liquid phase is responsible for the build-up of a solute boundary layer [13]. In order to better estimate the impact of the vibrations on the local flow in the vicinity of the growth interface, we plot the vertical profiles of the horizontal and vertical velocity components close to the right cold end wall at $x=3.79$ in Fig. 3. These profiles are obtained for $A=4, \operatorname{Pr}=0.0113, \mathrm{Gr}=200$, and either $\mathrm{Gr}_{\mathrm{v}}=0$ or $\mathrm{Gr}_{\mathrm{v}}=5000$ for $\alpha=0,45$, and $90^{\circ}$. From Fig. 3, it is clear that the velocity profiles close to the interface can be strongly changed by applying vibrations. In the case $\alpha=$ $0^{\circ}$, however, the vibrations, which are parallel to applied temperature gradient, have very little influence on the velocity profiles, which remain very close to those obtained without vibrations $\left(\mathrm{Gr}_{\mathrm{v}}=0\right)$. In the case $\alpha=90^{\circ}$, the amplitude of the velocity profiles is increased, but we can also note that the shape of the profiles is changed. This is due to the formation of a counterrotating cell located near the growth interface above the main convective cell, as already observed in Bouarab et al. [25]. Finally, it is the case at $\alpha=45^{\circ}$ which really gives strong increases of the velocity profiles. These profiles have the same shapes as for $\mathrm{Gr}_{\mathrm{v}}=0$, indicating a similar global convective cell in the cavity, but have strongly increased in amplitude. Therefore, the case $\alpha=45^{\circ}$ appears to be the most suitable to remove impurities away from 
the interface and prevent the build-up of a solute boundary layer. It could be a good candidate for the optimization of the purification process of metallurgical grade silicon.

The vibration angles close to 45 degrees were already mentioned in [24-25] to be optimal to get maximum flows in the cavity. It was also proved analytically by the creeping flow method in [27], as indicated in [18]. In order to theoretically assess this optimal vibration angle in our situation, we will follow the approach used to find expressions for the critical vibration angles $\alpha_{1}$ and $\alpha_{2}$ between which flow inversion occurs [25]. This optimal vibration angle will correspond to the maximum driving force in the cavity (or maximum driving torque, as expressed in Eq. (1)). We consider moderate flows of low Prandtl number fluids in rather long cavities $(A \geq 2$, as $A=4$ ) where a quite uniform flow exists in the central part away from the ends. In these conditions, as shown in Fig. 4(a) for $A=4, \mathrm{Pr}=0.01, \mathrm{Gr}=2500, \mathrm{Gr}_{\mathrm{v}}=6250$, and $\alpha=45^{\circ}$, we will have a close to diffusive temperature field in the cavity corresponding here to

$$
\frac{\partial T}{\partial x} \approx-1 \text { and } \frac{\partial T}{\partial z} \approx 0
$$

Moreover, as seen in Fig. 4(b), the stream function $F$ associated with the pulsation velocity $\boldsymbol{W}$ is quite uniform in the central part of the cavity with only a dependence along $z$, the variation with $x$ only occurring in the end parts. In the central part, Eq. (4) defining $F$ is then reduced to

$$
\frac{\partial^{2} F}{\partial z^{2}}=\left(\frac{\partial T}{\partial x}\right) \sin \alpha_{V}
$$

In such a long cavity, the driving torque is mainly that existing in the central part, the end zones allowing recirculation. Using $\partial T / \partial z \approx 0$ and the properties of $F$, the absolute torque $\Gamma$ in the central part can then be written as

$$
\Gamma=\left|\operatorname{Gr} \frac{\partial T}{\partial x}+\mathrm{Gr}_{\mathrm{v}}\left[\left(\frac{\partial^{2} F}{\partial z^{2}} \frac{\partial T}{\partial x}\right) \cos \alpha_{V}\right]\right|=\left|\mathrm{Gr} \frac{\partial T}{\partial x}+\mathrm{Gr}_{\mathrm{v}}\left(\frac{\partial T}{\partial x}\right)^{2} \sin \alpha_{V} \cos \alpha_{V}\right| .
$$

Finally using $\partial T / \partial x \approx-1$ and $\alpha=\pi-\alpha_{V}$, we get:

$$
\Gamma=\left|\mathrm{Gr}+\mathrm{Gr}_{\mathrm{v}} \sin \alpha \cos \alpha\right|=\left|\mathrm{Gr}+\frac{\mathrm{Gr}_{\mathrm{v}}}{2} \sin 2 \alpha\right|
$$

The maximum absolute torque will then be obtained for $\sin 2 \alpha=1$, i.e. for $\alpha=\pi / 4=45^{\circ}$. The results shown in [25] confirm well that the optimal vibration angle is close to $45^{\circ}$ for moderate flows corresponding to moderate values of $\mathrm{Gr}$ and $\mathrm{Gr}_{\mathrm{v}}$, and that it deviates from $45^{\circ}$ for strong flows, obtained for example for large values of $\mathrm{Gr}_{\mathrm{v}}$.

In the next section, through numerical simulations of the global solidification process, we will directly check the influence of these flows obtained under vibrations on the segregation of impurities in both the melt and the crystal.

\subsection{Effect of vibrations on silicon purification}

\subsubsection{Impurity concentration and solutal boundary layer}

Phosphorus, aluminum, and iron are considered to be among the main impurities in metallurgical grade silicon and consequently their presence in the grown silicon ingots reduces the energy conversion efficiency of the photovoltaic panels. We will then focus our study on the segregation of these impurities in silicon, during horizontal Bridgman solidification processes characterized by a pulling rate $v_{f}=-0.2$ (the typical value chosen by Kaddeche $e t$ al. [14]) and a Prandtl number $\operatorname{Pr}=0.0113$. The segregation coefficient $k$ and the Schmidt number Sc for these impurities are given in Table 1. Their initial concentration will be chosen 
as $C_{0}=1$. We will first consider phosphorus, which is characterized by a segregation coefficient $k=0.332$ and a Schmidt number Sc $=12.74$. Note that phosphorus is more difficult to remove from the molten silicon than the metallic impurities, which have small segregation coefficients.

The effect of vibrations on the segregation of phosphorus during directional solidification is illustrated in Fig. 5. These results, obtained for the optimal vibration direction $\left(\alpha=45^{\circ}\right)$ and $\mathrm{Gr}=200$, give the concentration of phosphorus in the melt (on the left-hand side) and in the crystal (on the right-hand side), when half of the sample has been solidified $(S=2)$ and for three values of the vibrational Grashof number, $\mathrm{Gr}_{\mathrm{v}}=0,1000$ and 5000 . Note first that, in all cases, due to the convective flow, the impurity concentration is weak in the core of the flow and larger in the vicinity of the melt/crystal interface inside the solute boundary layer. The direction of the flow (downwards along the interface) makes this boundary layer thicker at the bottom, with larger concentrations, which also gives crystals with larger concentrations at the bottom. The increase of $\mathrm{Gr}_{\mathrm{v}}$ induces an enhancement of the convective level due to the vibration of the crucible. The impurities rejected by the crystal are then better moved away from the interface and mixed in the core. A decrease of the larger phosphorus concentrations in the melt and in the crystal is then observed and this trend is more pronounced for $\mathrm{Gr}_{\mathrm{v}}=5000$, when the mixing is stronger (Fig. 5).

A key parameter to assess the interaction between convection and segregation is the solutal boundary layer thickness $\delta$ at the solid-liquid interface. Following Garandet et al. [28], the solutal boundary layer thickness is defined as $\left(C_{I}-C_{\infty}\right) /(\partial C / \partial z)_{I}$, where $C_{I}$ and $(\partial C / \partial z)_{I}$ are the concentration and concentration gradient at the interface and $C_{\infty}$ is the concentration far from the interface, taken in practice as the concentration at the left hot end wall. $\delta$ is then obtained as the average of the values calculated for all the discretization points along the interface. The effect of the vibrations on $\delta$ for the main impurities in PV silicon, namely phosphorus, aluminum, and iron, is illustrated in Fig. 6. The values of $\delta$, computed when half of the initial melt has been solidified, are given as a function of $\mathrm{Gr}_{\mathrm{v}}$ in the range $0 \leq \mathrm{Gr}_{\mathrm{v}} \leq$ 18000 and for $\alpha=45^{\circ}$. High frequency vibrations of the crucible lead to a strong decrease of the solutal boundary layer thickness $\delta$. The initial decrease of $\delta$, when $\mathrm{Gr}_{\mathrm{v}}$ is increased from 0 (in the range $0 \leq \mathrm{Gr}_{\mathrm{v}} \leq 2000$, for example), is particularly important. This decrease then slows down and seems to tend towards an asymptotic behavior corresponding to a very weak decrease. As shown in Fig. 6, the values of $\delta$ are very close for iron and aluminum, especially for large values of $\mathrm{Gr}_{\mathrm{v}}$. This is mainly due to their mass diffusivities in molten silicon, which are very close, giving close Schmidt numbers (Sc $=4.32$ for aluminum and Sc $=3.68$ for iron). Vibrations are then expected to have the same effect on the purification of metallurgical grade silicon for iron and aluminum impurities. Concerning phosphorus, its larger Schmidt number (Sc $=12.74$ ) leads to smaller values of $\delta$, which also decrease when $\mathrm{Gr}_{\mathrm{v}}$ is increased.

\begin{tabular}{|c|c|c|}
\hline & Sc & $k$ \\
\hline Phosphorus & 12.74 & 0.332 \\
\hline Aluminum & 4.32 & $2 \times 10^{-3}$ \\
\hline Iron & 3.68 & $8 \times 10^{-6}$ \\
\hline
\end{tabular}

Table 1: Schmidt number Sc and segregation coefficient $k$ for some of the main impurities in silicon.

\subsubsection{Axial segregation}


In order to investigate the influence of the vibrations on the impurity segregation in the crystal, we have computed the axial segregation $C_{s, a v}$ given by the following expression,

$$
C_{s, a v}=\int_{0}^{1} C_{s}(z, S) d z .
$$

$C_{S, a v}$ is averaged on the height of the crystal and calculated for each position $S$ of the interface from $S=A=4$ to $S=A / 2=2$. In the figures, it will be expressed as a function of the dimensionless solidified length $l_{s}$, i.e. for $l_{s}$ between 0 and 2 . Note that we have the relation $S+l_{s}=A$ between the dimensionless liquid and solid lengths.

The axial segregation of phosphorus is given in Fig. 7(a) for different values of $\mathrm{Gr}$ and $\mathrm{Gr}_{\mathrm{v}}$ $\left(\alpha=45^{\circ}\right)$. In all cases, due to the reject of impurity at the interface and the build-up of a solutal boundary layer, the axial segregation increases along the solidifying crystal. The increase is strong in a first stage (initial transient) and then smaller in a second stage corresponding to a more stationary regime [12]. This stationary regime can be better observed in Fig. 7(b) where, for the same cases as in Fig. 7(a), the solutal boundary layer thickness $\delta$ is given during the solidification process as a function of the solidified length $l_{s}$. Indeed, we see that, after an initial transient, $\delta$ becomes constant, this regime being obtained sooner for small $\delta$, i.e. for large convective effects. According to Fig. 7(a), more intense melt motion, due either to an increase of $\mathrm{Gr}$ or to a larger vibration effect, leads to smaller segregation levels, as found in previous works [12-15]. Note also that, when vibrations are applied in the optimal direction with a sufficient intensity, the amount of impurities in a case of rather weak buoyancy $(\mathrm{Gr}=200)$ can be similar to that obtained without vibration in a case with large buoyancy effect $(\mathrm{Gr} \sim 2000-$ 3000). Therefore, the enhancement of the convective level obtained by applying suitable vibrations is able to produce valuable purification effects. Comparing Fig. 7(a) and Fig. 7(b), we see the good correlation between the curves of axial segregation $C_{s, a v}$ and solutal boundary thickness $\delta$, the decrease of $\delta$ for increasing $\mathrm{Gr}_{\mathrm{v}}$ being well associated with smaller impurity concentrations, i.e. a better purification effect.

An analytic formula, derived by Scheil and Gulliver and known as the Scheil-Gulliver model, gives the impurity distribution along the solid length during solidification. The model assumes that the solute transfer in the melt is mainly achieved by convection (well-mixed regime). First formulated by Gulliver [29] and then solved by Scheil [30], the model gives

$$
C_{s}=k C_{0}\left(1-f_{s}\right)^{k-1},
$$

where $f_{s}$ is the solidified fraction given by $f_{s}=l_{s} / A$. An effective segregation coefficient $k_{e f f}=k /(1-(1-k) \Delta)$ can also be introduced in the Scheil-Gulliver model, in place of the equilibrium coefficient $k$, to account for the contribution of both diffusion and convection in the solute segregation and get a more realistic analytic law for axial segregation. The model is then denoted as the modified Scheil-Gulliver model. $\Delta$ used in the expression of $k_{\text {eff }}$ is the convecto-diffusive coefficient (normalized solutal boundary layer thickness) defined as $\Delta=$ $\delta V_{f} / D$. The effective segregation coefficient $k_{\text {eff }}$ reduces to $k$ when melt convection is intense, i.e. when $\Delta$ (or equivalently $\delta$ ) is very small. In contrast, in purely diffusive conditions, $\Delta$ and $k_{\text {eff }}$ are equal to unity.

As the modified Scheil-Gulliver model does not include the initial transient, the comparison of our numerical results with those provided by this model can only be effective during the stationary regime. Figure 7 shows a fairly good agreement between our computed axial segregation curves and those given by the modified Scheil-Gulliver model, especially when the 
convection level is high, i.e. for large $\mathrm{Gr}$ values or for large $\mathrm{Gr}_{\mathrm{v}}$ values associated with vibrations in the optimal direction $\left(\alpha=45^{\circ}\right)$.

The effect of the vibration direction on the axial segregation $C_{s, a v}$ is illustrated in Fig. 8 for $\mathrm{Gr}=200, \mathrm{Gr}_{\mathrm{v}}=5000$, and three vibrations angles $\left(\alpha=0^{\circ}, 45^{\circ}, 90^{\circ}\right)$. These cases are compared with the case without vibration $\left(\mathrm{Gr}_{\mathrm{v}}=0\right)$. The different vibration directions give clearly different segregation results. In the case of horizontal vibrations $\left(\alpha=0^{\circ}\right)$, no significant change in the segregation is found compared with the case without vibrations, in agreement with the almost not changed velocity field (Fig. 3). The vertical vibrations $\left(\alpha=90^{\circ}\right)$ give a clear global decrease of the axial segregation curves, but the regime is still not a well-mixed regime, as shown by the still rather bad agreement with the modified Scheil-Gulliver model. Finally, vibrations in the oblique direction at $\alpha=45^{\circ}$ still decrease the impurity concentration level in the solidified ingot. Moreover, the axial segregation curve now quite well fits with the modified Scheil-Gulliver model, indicating a well-mixed regime. We then confirm that this vibration direction is particularly interesting in view of optimizing silicon purification.

\subsubsection{Global impurity levels and limit of purification}

In this section, we will consider the effect of vibrations on the global impurity levels in the solid and liquid phases when half of the initial molten silicon is solidified. We will then define $C_{s, t o t}$ and $C_{l, t o t}$, which correspond to the integration of the impurity concentration in the solid and liquid phases, respectively. $C_{l, t o t}$ is then given by

$$
C_{l, t o t}=\int_{0}^{S=2} \int_{0}^{1} C(z, x) d z d x
$$

$C_{s, t o t}$ is obtained similarly by integration of $C_{s}$ in the solid zone with a length $l_{s}=2$. As the initial concentration of the melt in the $A=4$ ingot is $C_{0}=1$, by conservation of the impurities during the solidification process we will have $C_{s, t o t}+C_{l, t o t}=4$.

We will consider both phosphorus and aluminum impurities (Table 1) in cases where $\mathrm{Gr}=200$ and $\mathrm{Gr}_{\mathrm{v}}$ is increased from 0 to $18000\left(\alpha=45^{\circ}\right)$. As shown in Fig. 9, for both impurities, the global impurity level in the solid $C_{s, t o t}$ decreases when $\mathrm{Gr}_{\mathrm{v}}$ is increased, first quite steeply and then more slowly in a seemingly asymptotic variation. Conversely, the global impurity level in the liquid $C_{l, t o t}$ will increase, with similar characteristics. The change of the characteristics in the variation of $C_{s, t o t}$ with $\mathrm{Gr}_{\mathrm{v}}$ indicates that there is a limit value of $\mathrm{Gr}_{\mathrm{v}}$ beyond which the purification process becomes less efficient. This might be due to the fact that the well mixed regime is reached at this limit value of $\mathrm{Gr}_{\mathrm{v}}$. Such a change towards a well-mixed regime, which is associated with axial segregation curves getting very close to Scheil's curves, has been observed to occur in the case without vibration for $\mathrm{Gr}>5000$, as referred in Kaddeche et al. [14].

\subsubsection{Effect of flow inversion on purification}

In the recent work of Bouarab et al. [25], the influence of the vibration direction on the convection in a $A=4$ cavity was studied in detail for vibration angles varying from 0 to $180^{\circ}$. It was pointed out that an inversion of the flow, here from clockwise (direction of the buoyant flow) to counterclockwise could occur between two critical vibration angles $\alpha_{1}$ and $\alpha_{2}$ when the vibrational Grashof number is sufficiently high. The condition for flow inversion to occur was given by

$\mathrm{Gr}_{\mathrm{v}}>2 \mathrm{Gr}$,

and theoretical expressions were derived for $\alpha_{1}$ and $\alpha_{2}$, 
$\alpha_{1}=90^{\circ}+\theta_{0} / 2$ and $\alpha_{2}=180^{\circ}-\theta_{0} / 2$ with $\theta_{0}=\operatorname{asin}\left[2 /\left(\mathrm{Gr}_{\mathrm{v}} / \mathrm{Gr}\right)\right]$,

which match very well with the numerical results obtained with a spectral collocation method for different values of the Grashof and vibrational Grashof numbers. These expressions have been derived assuming that flow inversion will occur when the global driving force (or driving torque) is zero in the central part of the cavity [25].

In this section, we want to see more precisely the influence of the vibration direction on the segregation, and in particular analyze the effect due to the flow inversion. For that, we first present results concerning phosphorus and obtained for $\mathrm{Gr}=200$, all the possible vibration directions $\left(0^{\circ} \leq \alpha \leq 180^{\circ}\right)$ and different values of $\mathrm{Gr}_{\mathrm{v}}$ ranging from 100 to 10000 . Figure 10 first depicts the global impurity level in the silicon ingot $C_{s, t o t}$ versus the vibration angle for the different values of $\mathrm{Gr}_{\mathrm{v}}$. The reference case without vibrations $\left(\mathrm{Gr}_{\mathrm{v}}=0\right)$ is given by a pink dashed line. The parts of the curves below this line indicate cases with vibration characteristics $\left(\mathrm{Gr}_{\mathrm{v}}, \alpha\right)$ allowing purification, whereas the parts of the curves above this line indicate cases which have to be avoided as they induce an increase of the impurity level in the ingot. We see that for vibrations angles in the range $0^{\circ} \leq \alpha \leq 90^{\circ}$, except very close to $0^{\circ}$ and $90^{\circ}$, vibrations induce a purification, which is improved when $\mathrm{Gr}_{\mathrm{v}}$ is increased. In contrast, in the range $90^{\circ} \leq$ $\alpha \leq 180^{\circ}$, the results are more complex and strongly evolve with $\alpha$ and $\mathrm{Gr}_{\mathrm{v}}$. For a better understanding of the results, we will divide the $\mathrm{Gr}_{\mathrm{v}}$ range in different domains according to the effect induced by vibrations. The effect obtained for weak $\mathrm{Gr}_{\mathrm{v}}$ is shown in Figs. 11 and 12, whereas the effect for large $\mathrm{Gr}_{\mathrm{v}}$ is shown in Figs. 13 and 14.

As the calculations have been done for $\mathrm{Gr}=200$, the condition (10) for flow inversion indicates that no flow inversion will occur for $\mathrm{Gr}_{\mathrm{v}} \leq 400$. The global impurity level versus $\alpha$ is shown for these cases in Fig. 11(a) and the corresponding variation of $\psi_{c}$, the stream function at the center of the melt zone when half of the silicon has been solidified $(S=2)$, in Fig. 11(b). As expected, $\psi_{c}$ keeps the same sign as the purely buoyant flow in the whole range of vibration angles, indicating the persistence of a clockwise flow. The intensity of this flow, however, depends on $\alpha$ and has a maximum for $\alpha \approx 45^{\circ}$ and a minimum for $\alpha \approx 135^{\circ}$. Note that the minimum of $\psi_{c}$ for $\mathrm{Gr}_{\mathrm{v}}=400$ is close to zero, indicating that it is well the limit case without inversion, as given by Eq. (10).

Concerning purification for these cases, as expected, purification is improved by the vibrations when the flow intensity is increased, i.e. in the range $0^{\circ}<\alpha<90^{\circ}$, whereas it is degraded when the flow intensity is decreased, i.e. in the range $90^{\circ}<\alpha<180^{\circ}$ (Fig. 11(a)). The benefit obtained by vibrations, however, remains limited for these weak $\mathrm{Gr}_{\mathrm{v}}$ values, even for the optimal directions (about $5 \%$ only for $\mathrm{Gr}_{\mathrm{v}}=400$ and $\alpha=45^{\circ}$ ). The degradation can be more significant, with an increase of $C_{s, t o t}$ up to $20 \%$ for $\mathrm{Gr}_{\mathrm{v}}=400$ and vibration angles in the vicinity of $\alpha=135^{\circ}$. These vibration angles have really to be avoided in the case of weak $\mathrm{Gr}_{\mathrm{v}}$ values. Finally, we see that the vibrations either parallel $\left(\alpha=0^{\circ}\right)$ or perpendicular $\left(\alpha=90^{\circ}\right)$ to the imposed temperature gradient have almost no effect on the purification. An illustration of the velocity and concentration fields in the cases with weak $\mathrm{Gr}_{\mathrm{v}}$ is given in Fig. 12 for the vibration angles giving maximal variations $\left(\alpha=45^{\circ}\right.$ and $135^{\circ}$ ). For $\mathrm{Gr}_{\mathrm{v}}=100$ (Fig. 12(a)), the effect on the flow and concentration fields remains moderate, and these fields keep a similar structure as in the reference case without vibrations $\left(\mathrm{Gr}_{\mathrm{v}}=0\right.$ ). For $\mathrm{Gr}_{\mathrm{v}}=400$ (Fig. 12(b)), the vibrations have a more noticeable effect. The more intense flow for $\alpha=45^{\circ}$ induces a concentration field with more deformed iso-concentration lines. In contrast, the less intense and modified flow structure for $\alpha=135^{\circ}$ gives a less perturbed concentration field, closer to diffusive conditions. 
For larger values of $\mathrm{Gr}_{\mathrm{v}}\left(\mathrm{Gr}_{\mathrm{v}}>400\right)$, the global impurity level in the silicon ingot $C_{s, t o t}$ versus the vibration angle $\alpha$ is depicted in Fig. 13. In all these cases ( $\mathrm{Gr}_{\mathrm{v}}$ ranging from 500 to 10000), the flow direction at the center of the melt is inverted in the $\left(90^{\circ}, 180^{\circ}\right) \alpha$ range between the values $\alpha_{1}$ and $\alpha_{2}$ depending on $\mathrm{Gr}_{\mathrm{v}}$ and given by (11). These limit values $\alpha_{1}$ and $\alpha_{2}$, where the flow is the weakest, correspond to peaks in the $C_{s, t o t}$ curves (maximum impurity level). Between $\alpha_{1}$ and $\alpha_{2}$, the inverted flow can develop, with a maximum intensity for $\alpha$ close to $135^{\circ}$. For values of $\mathrm{Gr}_{\mathrm{v}}$ far above 400, this inverted flow can be sufficiently strong to also induce purification. In Fig. 13, we can see that this is the case for $\mathrm{Gr}_{\mathrm{v}}=2000,5000$, and 10000 , where $C_{s, t o t}$ around $\alpha=135^{\circ}$ is below the value obtained without vibration $\left(\mathrm{Gr}_{\mathrm{v}}=0\right)$. For large values of $\mathrm{Gr}_{\mathrm{v}}$ as $\mathrm{Gr}_{\mathrm{v}}=10000$, purification of silicon can be obtained as well with the normal flow around $\alpha=45^{\circ}$ as with the inverted flow around $\alpha=135^{\circ}$, and with a similar efficiency. This is consistent with the fact that, as shown by Bouarab et al. [25], in the limit of large $\mathrm{Gr}_{\mathrm{v}} / \mathrm{Gr}$, the flow obtained for $\alpha=90^{\circ}+\theta$ is exactly the reverse flow of that obtained for $\alpha=90^{\circ}-\theta$ and corresponds to its symmetric with respect to the up-down symmetry. Note that, for these large values of $\mathrm{Gr}_{\mathrm{v}}$, the minima of $C_{s, t o t}$, corresponding to the optimal purification directions, are shifted from 45 and $135^{\circ}$ to 30 and $150^{\circ}$, respectively. In fact, large ranges of vibration angles can be used for purification in these cases, except $\alpha$ values close to 0,90 , and $180^{\circ}$, which correspond to vibrations directions parallel or perpendicular to the imposed temperature gradient.

We will now focus in more details on the case corresponding to $\mathrm{Gr}_{\mathrm{v}}=5000$, for which are given in Fig. 14 the $\psi_{c}$ and $C_{s, t o t}$ curves versus $\alpha$ and different flow and concentration fields. For this case $\left(\mathrm{Gr}=200, \mathrm{Gr}_{\mathrm{v}}=5000\right)$, the analytic expression (11) of the critical angles for flow inversion gives $\alpha_{1}=92.29^{\circ}$ and $\alpha_{2}=177.71^{\circ}$. These are exactly the values found by the numerical calculations for a cavity with an aspect ratio $A=4$, i.e. at the beginning of the solidification process. For $S=2$, i.e. when half the sample has been solidified (Fig. 14), the values obtained numerically change slightly to $\alpha_{1}=92.66^{\circ}$ and $\alpha_{2}=177.43^{\circ}$. This is due to the fact that some hypotheses on the temperature $T$ and the pulsation velocity stream function $F$ used to derive the analytical expressions are rather valid in long cavities where a clear quiescent central zone can appear. The discrepancy for $S=2$, however, remains very weak. In any case, the $\alpha$ range of flow inversion is large in this case due to the already strong $\mathrm{Gr}_{\mathrm{v}} / \mathrm{Gr}$ ratio $\left(\mathrm{Gr}_{\mathrm{v}} / \mathrm{Gr}=25\right)$. This $\alpha$ range is close to the limit $\left[90^{\circ}, 180^{\circ}\right]$ obtained for very large $\mathrm{Gr}_{\mathrm{v}} / \mathrm{Gr}$ ratios.

Figure 14 gives interesting information on the effect of the vibrations direction on the global impurity level in the ingot when half of the melt has been solidified. In the case without vibrations, $C_{s, t o t}$ is equal to 1.17 , value given by a horizontal pink dashed line. For most vibrations directions, the $C_{s, t o t}$ values are below this line, indicating an improved purification effect. This effect is obtained in a large $\alpha$ range around $45^{\circ}$ with the normal flow, but also in a large $\alpha$ range around $135^{\circ}$ with the reverse flow. If we compare the cases at $\alpha=45^{\circ}$ and $135^{\circ}$, we see that the global impurity levels given by $C_{s, t o t}$ are comparable (although still a little stronger for $\alpha=135^{\circ}$ ), but, as shown by the flow and concentration fields given as insets, they are obtained by flows rotating in opposite directions. More precisely, as expected for large $\mathrm{Gr}_{\mathrm{v}} / \mathrm{Gr}$ ratios [25], there is almost an up-down symmetry between the fields at $\alpha=45^{\circ}$ and $135^{\circ}$, and as a result, the ingot is enriched at its bottom for $\alpha=45^{\circ}$ and at its top for $\alpha=135^{\circ}$. Finally, the vibration angles close to $\alpha_{1}$ and $\alpha_{2}$, i.e. close to 0,90 , and $180^{\circ}$ for these large $\mathrm{Gr}_{\mathrm{v}} / \mathrm{Gr}$ ratios, have to be avoided as they give a maximum amount of impurity, due to a low 
level of convection. However, as illustrated in the insets in Fig. 14 and in agreement with what is shown in Bouarab et al. [25], the flow patterns for $\alpha_{1}$ and $\alpha_{2}$ (which both corresponds to $\psi_{c}=0$ ) are completely different. For $\alpha_{1}=92.66^{\circ}$, the flow pattern consists of four vortices with two counter-rotating vortices at each side of the melt domain, in particular along the interface. This flow has almost an up-down symmetry, which is also observed in the concentration fields in the melt and in the ingot. For $\alpha_{2}=177.43^{\circ}$, the flow pattern consists of two co-rotating vortices with a stagnation point between them, which gives a single vortex along the interface. These differences in the flow structure lead to significant differences in the impurity concentration fields in both the molten silicon and the ingot. The global impurity level is also different, more important for $\alpha_{2}\left(C_{s, t o t}=1.37\right)$ than for $\alpha_{1}\left(C_{s, t o t}=1.19\right)$.

Finally, for the extremum values of $C_{s, t o t}$, corresponding to vibration angles $\alpha$ close to $45^{\circ}$, $92.66^{\circ}\left(\alpha_{1}\right), 135^{\circ}$, and $177.43^{\circ}\left(\alpha_{2}\right)$ (see the insets in Fig. 14), we give the axial segregation curves in Fig. 15. We see that the directions $\alpha=45^{\circ}$ and $135^{\circ}$ give almost the same averaged impurity concentration $C_{s, a v}$ along the ingot, strongly improved with respect to the case without vibrations $\left(\mathrm{Gr}_{\mathrm{v}}=0\right)$. These two directions of vibrations (as well as the neighboring directions) can be recommended as the optimal directions for purifying the metallurgical grade silicon, assuming the $\mathrm{Gr}_{\mathrm{v}} / \mathrm{Gr}$ ratio is large. If this condition is not fulfilled, only the directions around $\alpha=45^{\circ}$ have to be used. Fig. 15 also shows that the directions $\alpha_{1}$ and $\alpha_{2}$ (and their neighborhood) have to be avoided as they do not lead to purification, particularly $\alpha_{2}$ which gives the higher impurity level along the ingot.

\section{Conclusion}

The use of high frequency vibrations applied to the crucible can be a relevant non-intrusive active control technique for purifying metallurgical grade silicon. The direction of the vibrations, however, is known to significantly change the flow intensity and structure in the melt and, as a consequence, can be expected to influence the impurity concentration in the melt and the final amount of impurities in the grown ingot.

The study was performed for a $A=4$ crucible with the solidification of half the initial melt. The influence of the vibrations on the impurities concentration in the melt and in the silicon ingot during the solidification was characterized for different intensities and directions of vibrations. It is shown that, for the different impurities, the purification of the silicon ingot can be improved by vibrations, provided that the vibration direction is judiciously chosen. The vibrations directions around $\alpha=45^{\circ}$ are found to be the optimal directions to improve the purification process, as they keep the one-roll structure of the buoyant flow, but optimally increase its intensity, this increase depending on the strength of the vibrational Grashof number $\mathrm{Gr}_{\mathrm{v}}$. For large values of $\mathrm{Gr}_{\mathrm{v}}$ with respect to the Grashof number $\mathrm{Gr}$ (large $\mathrm{Gr}_{\mathrm{v}} / \mathrm{Gr}$ ratios), the vibrations directions around $\alpha=135^{\circ}$ are also efficient directions for the purification, as they induce reverse flows with respect to the buoyant flow, but with an intensity comparable to that of the flow obtained for $\alpha=45^{\circ}$. In contrast, the vibrations directions $\alpha_{1}$ and $\alpha_{2}$, which correspond to the transition between the normal flow and the reverse flow, have to be avoided, as they give weak flows and bad results in terms of purification. These critical vibrations directions $\alpha_{1}$ and $\alpha_{2}$ can still be accurately obtained by the analytical expressions proposed by Bouarab et al. [25] for a long $A=4$ cavity, despite the fact that the melt zone decreases in size during the solidification process.

\section{Acknowledgements}


This collaborative work was supported by the PHC Maghreb Partnership Program no. 36951NG. A grant of the PROFAS B+ program given to A.M. is also gratefully acknowledged.

\section{References}

1. A. Goetzberger, C. Hebling, H.W. Schock, Photovoltaic materials, history, status and outlook, Materials Science and Engineering 40 (2003) 1-46.

2. S. Pizzini, Towards solar grade silicon: challenges and benefits for low cost photovoltaics, Solar Energy Materials and Solar Cells 94 (2010) 1528-1533.

3. Y. Delannoy, Purification of silicon for photovoltaic applications, Journal of Crystal Growth 360 (2012) 61-67.

4. B. Gao, X.J. Chen, S. Nakano, K. Kakimoto, Crystal growth of high purity multicrystalline silicon using a unidirectional solidification furnace for solar cells, Journal of Crystal Growth 312 (2010) 1572-1576.

5. S. Dumitrica, D. Vizman, J. P. Garandet, A. Popescu, Numerical studies on a type of mechanical stirring in directional solidification method of multicrystalline silicon for photovoltaic applications, Journal of Crystal Growth 360 (2012) 76-80.

6. M. Chatelain, V. Botton, M. Albaric, D. Pelletier, B. Cariteau, D. Abdo, M. Borrelli, Mechanical stirring influence on solute segregation during plane front directional solidification, International Journal of Thermal Sciences 126 (2018) 252-262.

7. K. Dadzis, D. Vizman, J. Friedrich, Unsteady coupled 3D calculations of melt flow, interface shape, and species transport for directional solidification of silicon in a traveling magnetic field, Journal of Crystal Growth 367 (2013) 77-87.

8. N. Dropka, C. Frank-Rotsch, P. Rudolph, Numerical study on stirring of large silicon melts by Carousel magnetic fields, Journal of Crystal Growth 354 (2012) 1-8.

9. P. Capper, E. Zharikov, Oscillatory-driven fluid flow control during crystal growth from the melt, in Bulk Crystal Growth (Handbook of Crystal Growth, Elsevier, P. Rudolph editor, $2^{\text {nd }}$ edition 2015) 951-993.

10. W. Dridi, D. Henry, H. Ben Hadid, Influence of acoustic streaming on the stability of melt flows in horizontal Bridgman configurations, Journal of Crystal Growth 310 (2008) 1546-1551.

11. N.S. Ganesan, S. Manickam, A. Karuppanan, R. Perumalsamy, Simulation analysis on impurity distribution in $\mathrm{mc}-\mathrm{Si}$ grown by directional solidification for solar cell applications, International Journal of Materials Research 107 (2016) 525-533.

12. J. P. Garandet, S. Corre, S. Kaddeche, T. Alboussière, The influence of convection on the duration of the initial solute transient in alloy crystal growth, Journal of Crystal Growth 209 (2000) 970-982.

13. J. P. Garandet, T. Duffar, J. J. Favier, On the scaling analysis of the solute boundary layer in idealized growth configurations, Journal of Crystal Growth 106 (1990) 437444.

14. S. Kaddeche, H. Ben Hadid, D. Henry, Macrosegregation and convection in the horizontal Bridgman configuration. I. Dilute alloys, Journal of Crystal Growth 135 (1994) 341-353.

15. S. Kaddeche, H. Ben Hadid, D. Henry, Macrosegregation and convection in the horizontal Bridgman configuration. II. Concentrated alloys, Journal of Crystal Growth 141 (1994) 279-290. 
16. J.I.D. Alexander, J. P. Garandet, J.J. Favier, A. Lizee, G-Jitter effects on segregation during directional solidification of tin-bismuth in the Mephisto furnace facility, Journal of Crystal Growth 178 (1997) 657-661.

17. X. Bai, B. Ban, J. Li, Z. Fu, Z. Peng, C. Wang, J. Chen, Segregation behavior of metal impurities during Al-Si melt directional solidification with an open ended crucible, Silicon 10 (2018) 1283-1290.

18. G.Z. Gershuni, D.V. Lyubimov, Thermal Vibrational Convection (Wiley \& Sons, New York, 1998).

19. G.Z. Gershuni, A.K. Kolesnikov, J.C. Legros, B.I. Myznikova, On the vibrational convective instability of a horizontal, binary-mixture layer with the Soret effect, Journal of Fluid Mechanics 330 (1997) 251-269.

20. R. Savino, R. Monti, M. Piccirillo, Thermovibrational convection in a fluid cell, Computers and Fluids 27 (1998) 923-939.

21. T.P. Lyubimova, D.V. Lyubimov, A.O. Ivantsov, The influence of vibrations on melt flows during detached Bridgman crystal growth, Journal of Crystal Growth 385 (2014) $77-81$.

22. A.N. Sharifulin, Controllable equilibrium of an inhomogeneously heated liquid in a vibrational field, Journal of Experimental and Theoretical Physics 110 (2010) 157-161.

23. M. Lappa, Patterning behavior of gravitationally modulated supercritical Marangoni flow in liquid layers, Physical Review E 93 (2016) 053107.

24. M. Lappa, Control of convection patterning and intensity in shallow cavities by harmonic vibrations, Microgravity Science and Technology 28 (2016) 29-39.

25. S. Bouarab, F. Mokhtari, S. Kaddeche, D. Henry, V. Botton, A. Medelfef, Theoretical and numerical study on high frequency vibrational convection: influence of the vibration direction on the flow structure, Physics of Fluids 31 (2019) 043605.

26. H. Ben Hadid, B. Roux, Macrosegregation during crystal growth in horizontal Bridgman boat, Advances in Space Research 8 (1988) 251-264.

27. A.N. Sharifulin, Vibrational convection in cylindrical cavity under arbitrary direction of heating, in Convective Flows (Perm, 1981) 22-29.

28. J. P. Garandet, J.J. Favier, D. Camel, Solutal boundary layer concept and scaling analysis: two keys to segregation phenomena in melt crystal growth, Journal of Crystal Growth 130 (1993) 113-122.

29. G.M. Gulliver, The quantitative effect of rapid cooling upon the constitution of binary alloys, J. Inst. Metals 9 (1913) 120-157.

30. E. Scheil, Bemerkungen zur Schichtkristallbildung, Z. Metallkd. 34 (1942) 70-72. 


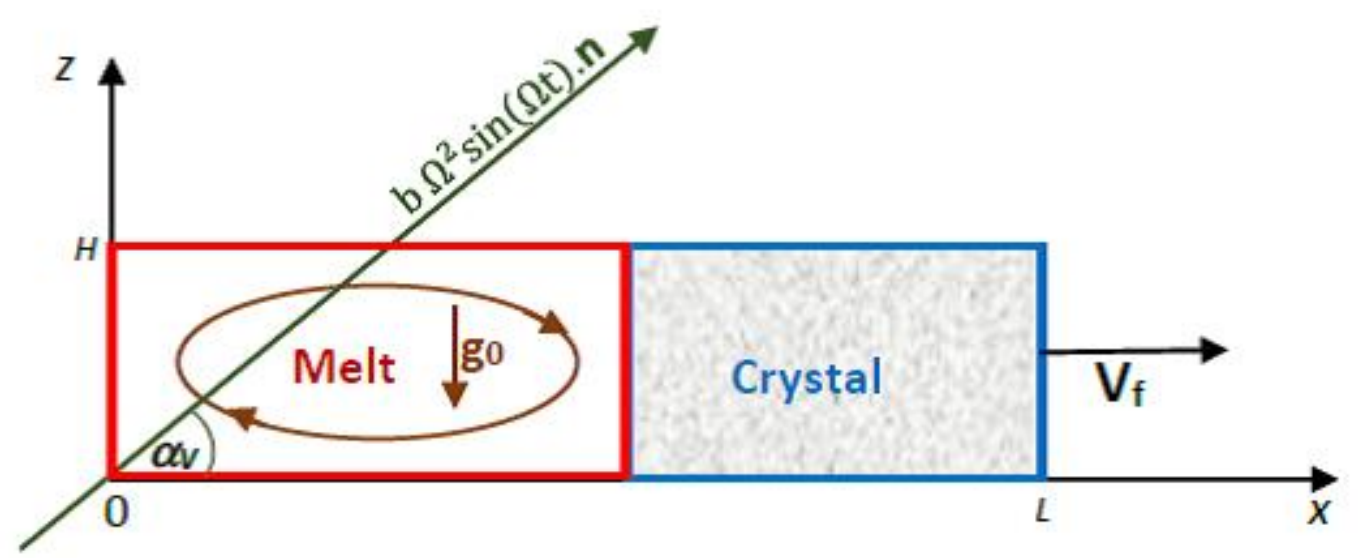

Fig. 1. Schematic of the horizontal Bridgman situation subject to vibrations.

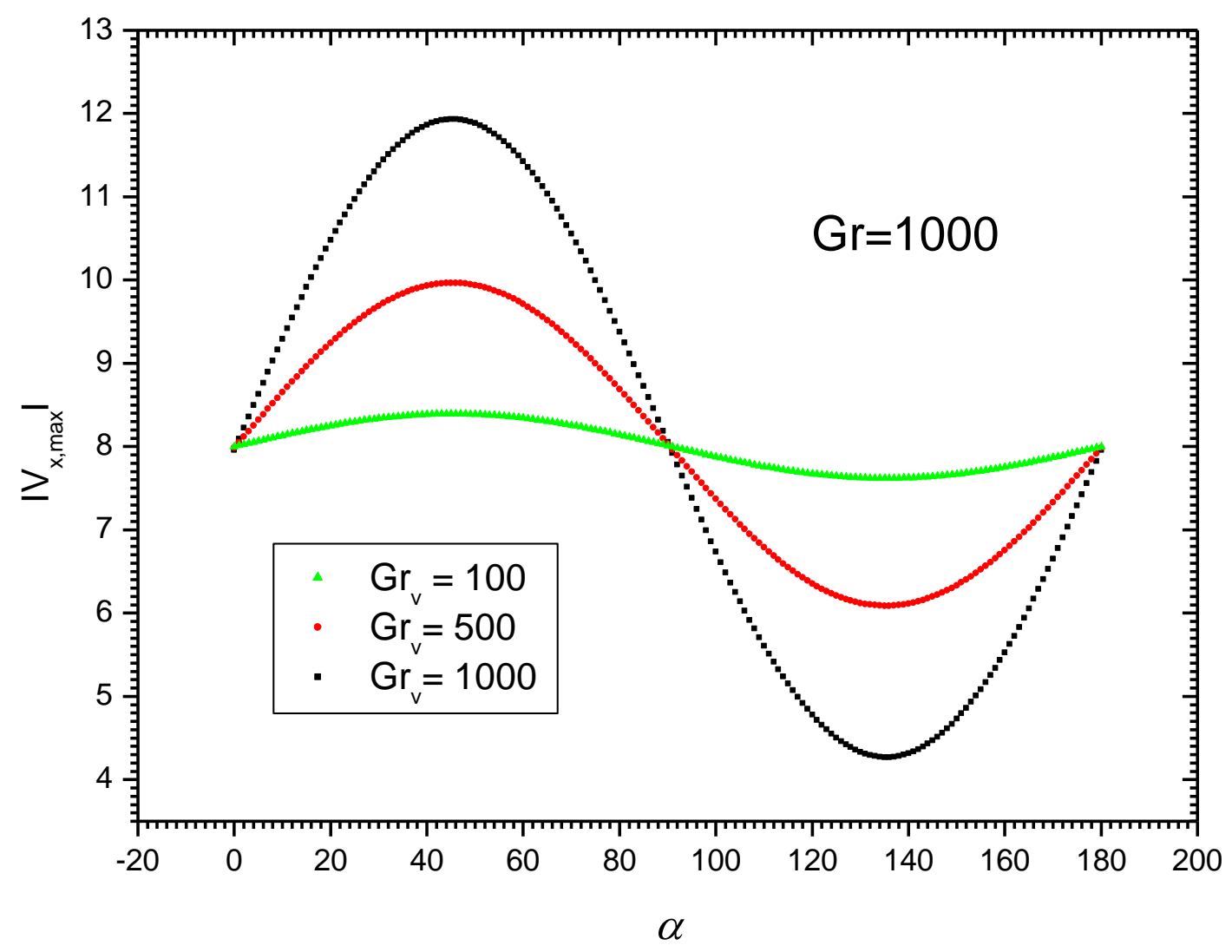


(a)

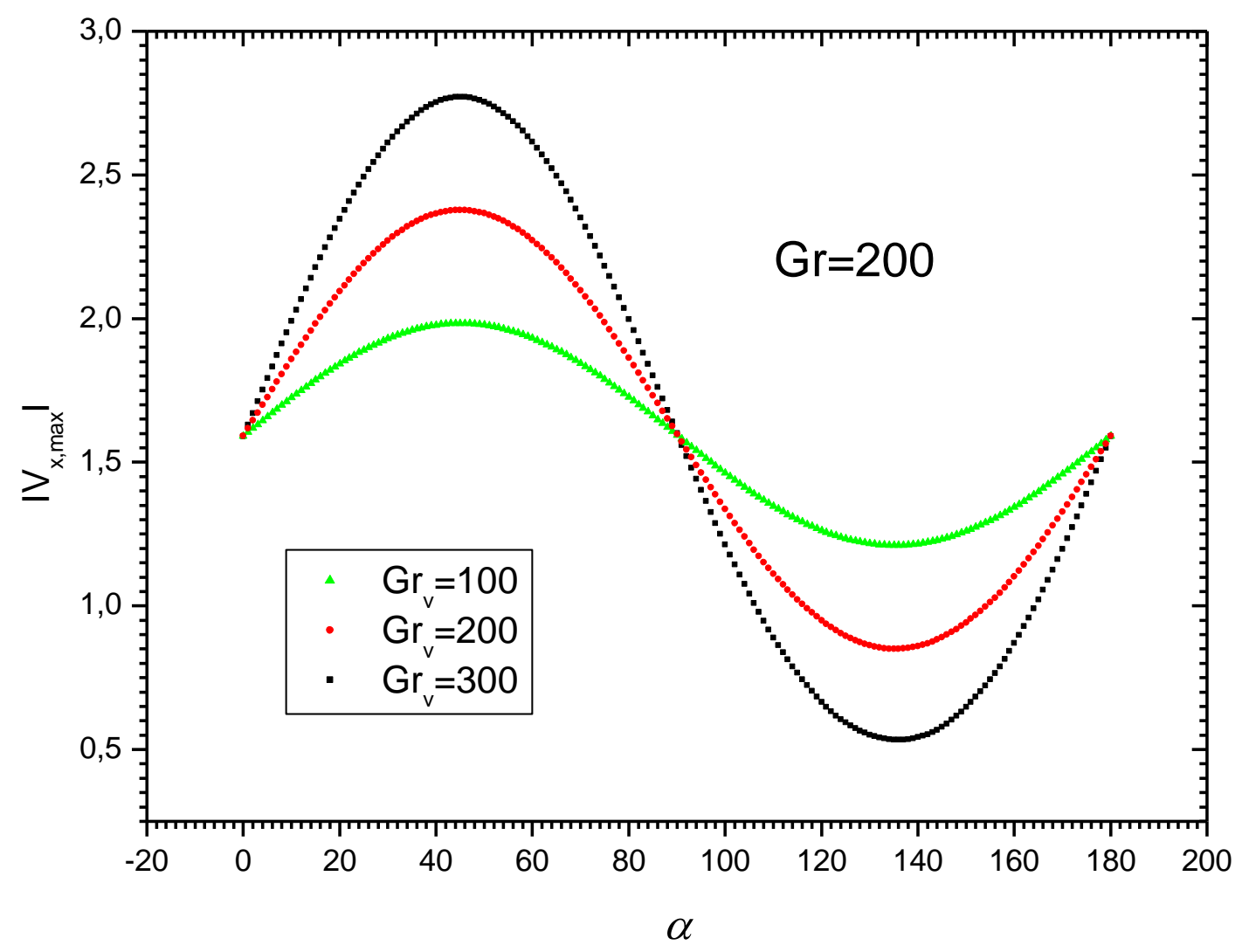

(b)

Fig. 2. Maximal horizontal velocity in the $A=4$ cavity as a function of the vibration angle $\alpha$ for $\mathrm{Gr}=1000$ (a) and $\mathrm{Gr}=200$ (b) and different values of $\mathrm{Gr}_{\mathrm{v}}(\mathrm{Pr}=0.0113)$. 


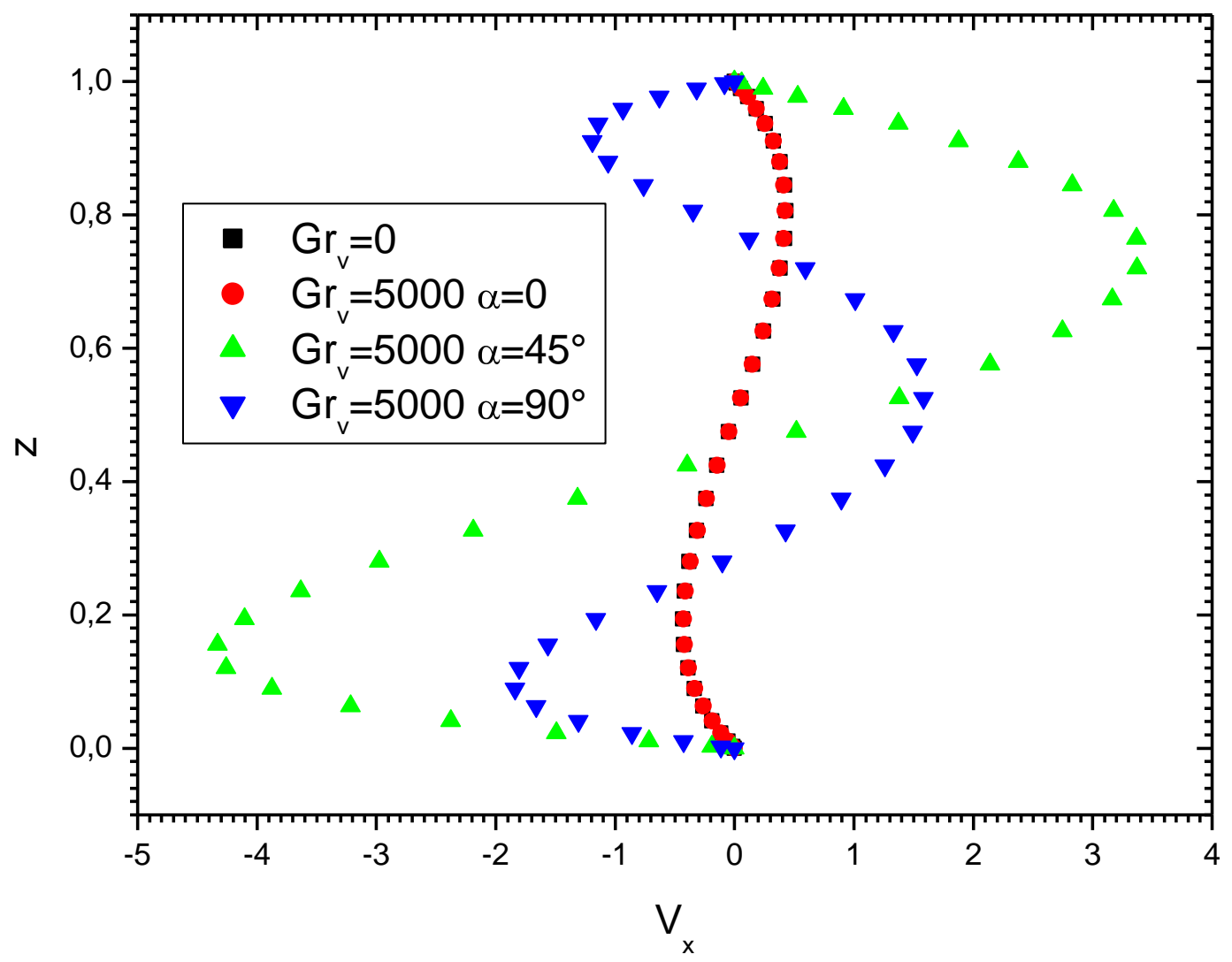

(a) 


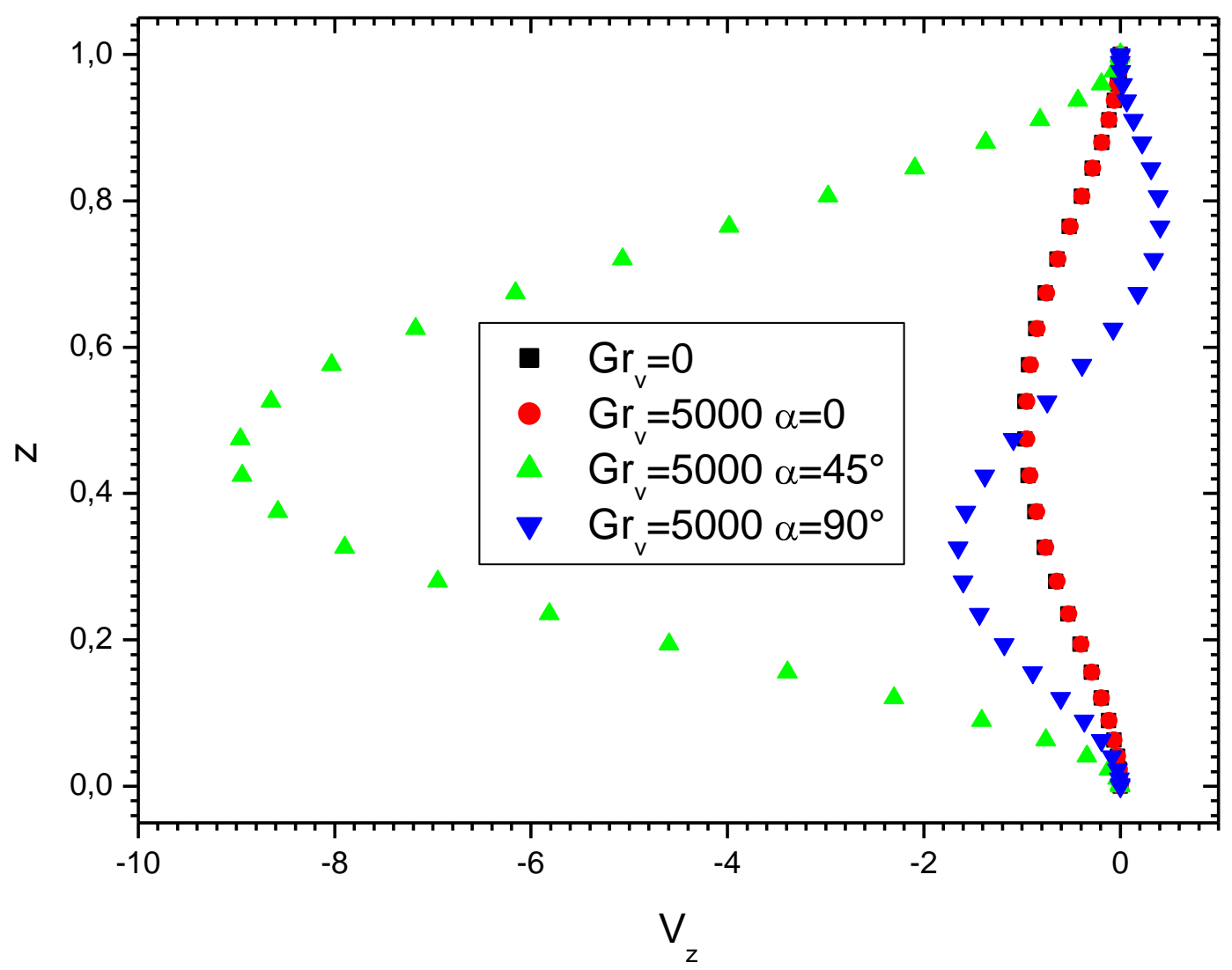

(b)

Fig. 3. Horizontal (a) and vertical (b) velocity profiles along $z$ close to the right cold end-wall at $x=3.79$, for $\mathrm{Gr}=200$, without vibration $\left(\mathrm{Gr}_{\mathrm{v}}=0\right)$ and with vibrations $\left(\mathrm{Gr}_{\mathrm{v}}=5000\right)$ applied in three different directions $\alpha=0,45$, and $90^{\circ}$. 


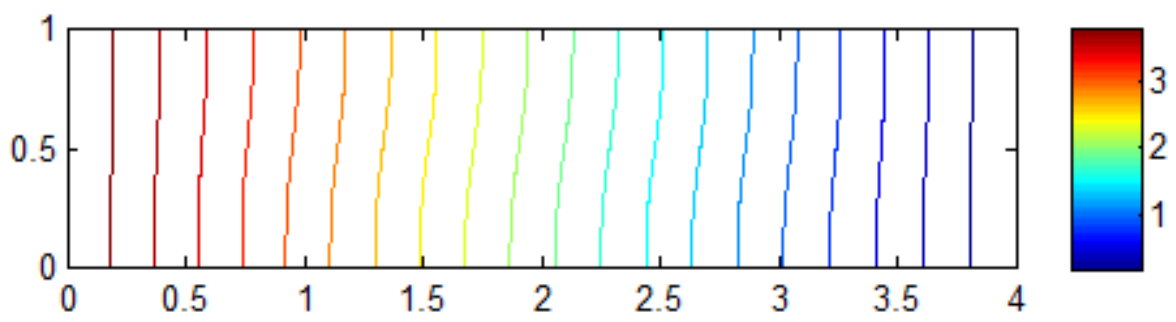

(a)

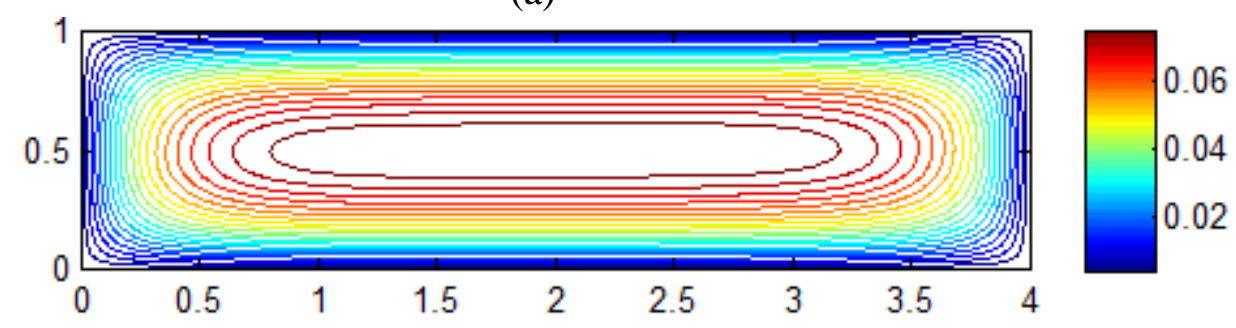

(b)

Fig. 4: Isotherms (a) and iso- $F$ lines (b) in a $A=4$ side-heated cavity submitted to vibrations for the case $\mathrm{Pr}=0.01, \mathrm{Gr}=2500$ and $\mathrm{Gr}_{\mathrm{v}}=6250$ with a vibration angle $\alpha=45^{\circ}$. Note that the isotherms are only weakly deformed, indicating that the hypothesis of a planar vertical interface makes sense. 


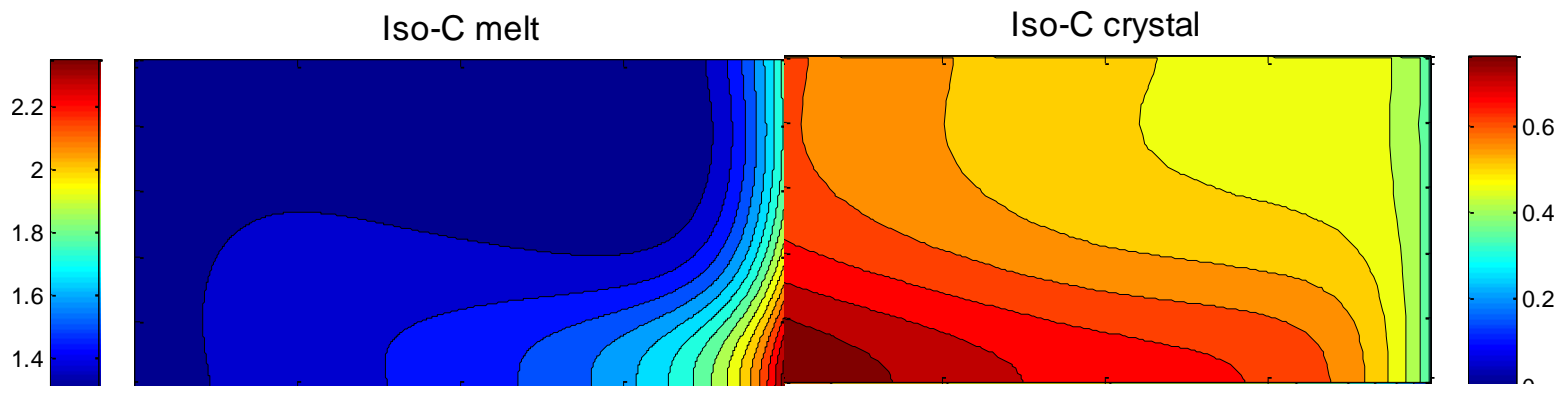

(a)

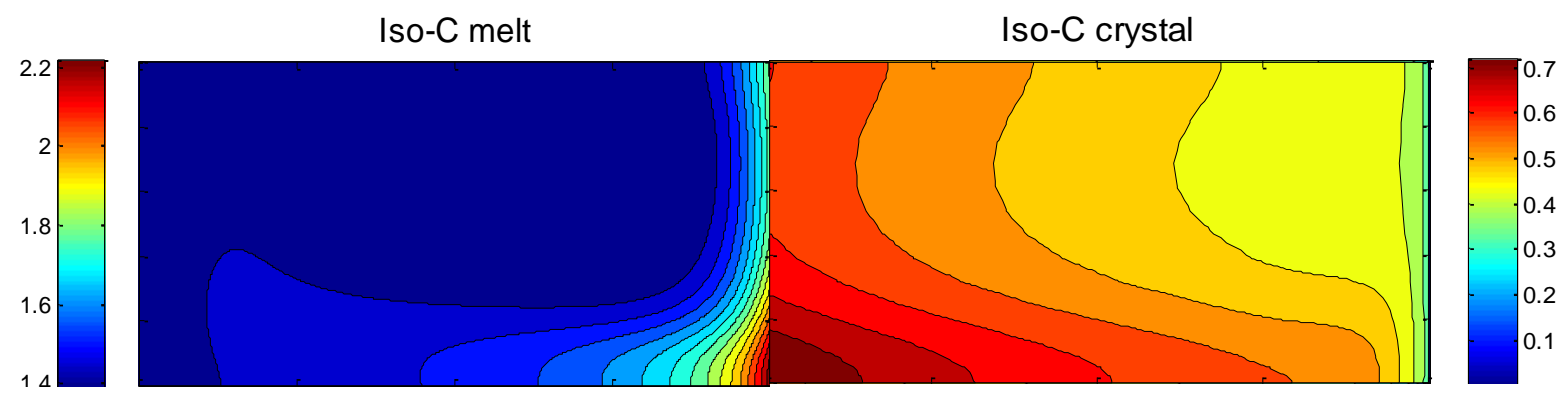

(b)

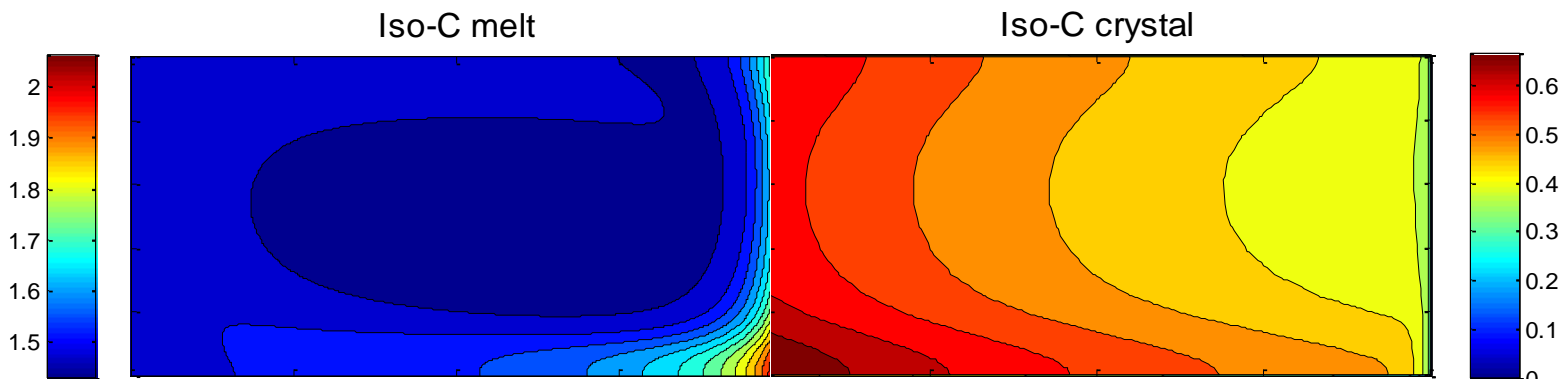

(c)

Fig. 5. Iso-concentration lines of phosphorus in the molten (left part) and solid (right part) silicon for $S=2, \operatorname{Pr}=0.0113, \mathrm{Gr}=200$, and different vibrational Grashof numbers: (a) $\mathrm{Gr}_{\mathrm{v}}=0$, (b) $\mathrm{Gr}_{\mathrm{v}}=1000$, and (c) $\mathrm{Gr}_{\mathrm{v}}=5000$, for $\alpha=45^{\circ}$. 


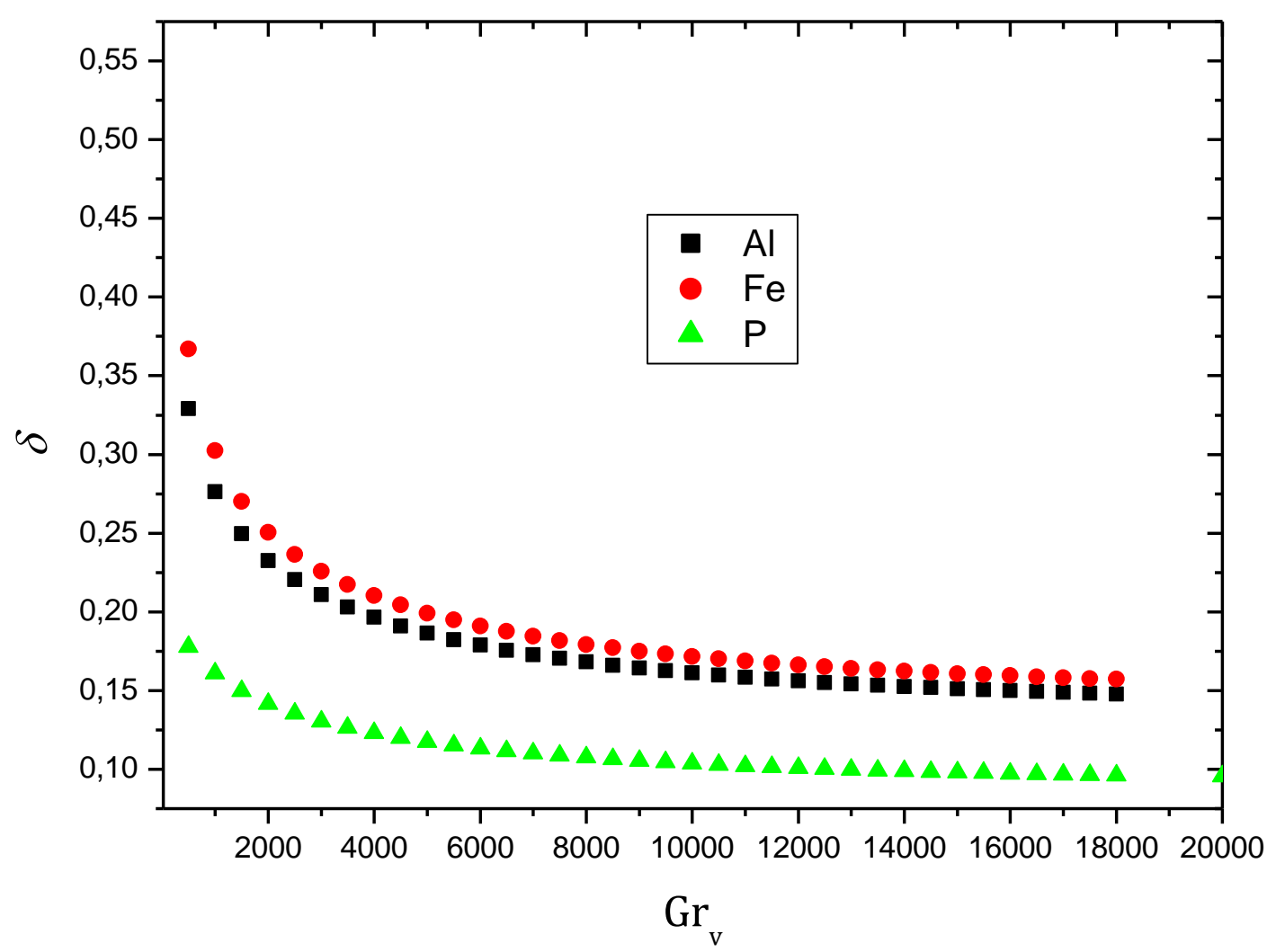

Fig. 6. Variation of the solute boundary layer thickness $\delta$ with the vibrational Grashof number $\mathrm{Gr}_{\mathrm{v}}$ for phosphorus $(\mathrm{Sc}=12.74)$, aluminum $(\mathrm{Sc}=4.32)$ and iron $(\mathrm{Sc}=3.68)$ impurities. The values are computed when half of the molten silicon has been solidified $(S=2)$ with vibrations applied in the optimal direction $\alpha=45^{\circ}(\mathrm{Gr}=200, \operatorname{Pr}=0.0113)$. 


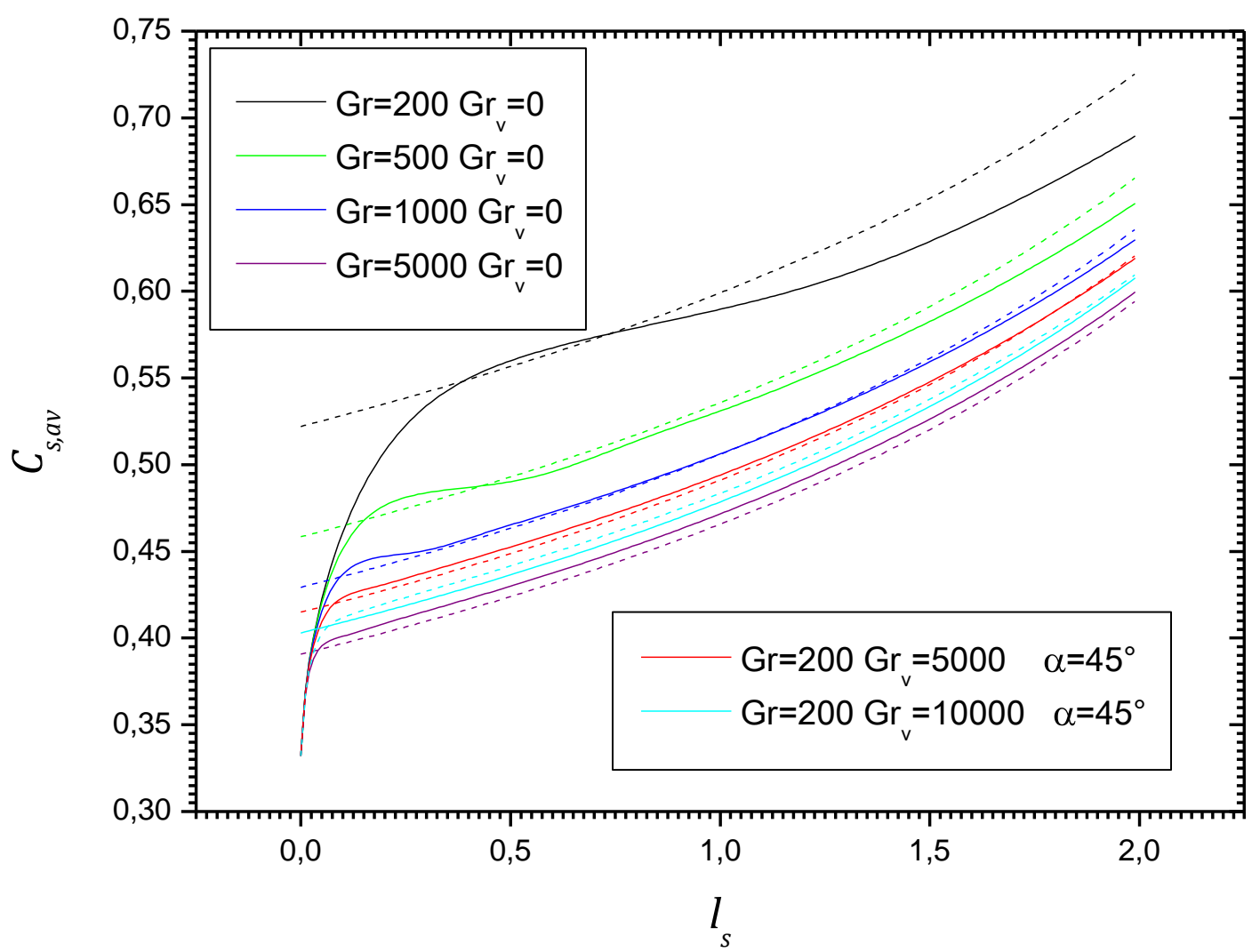

(a)

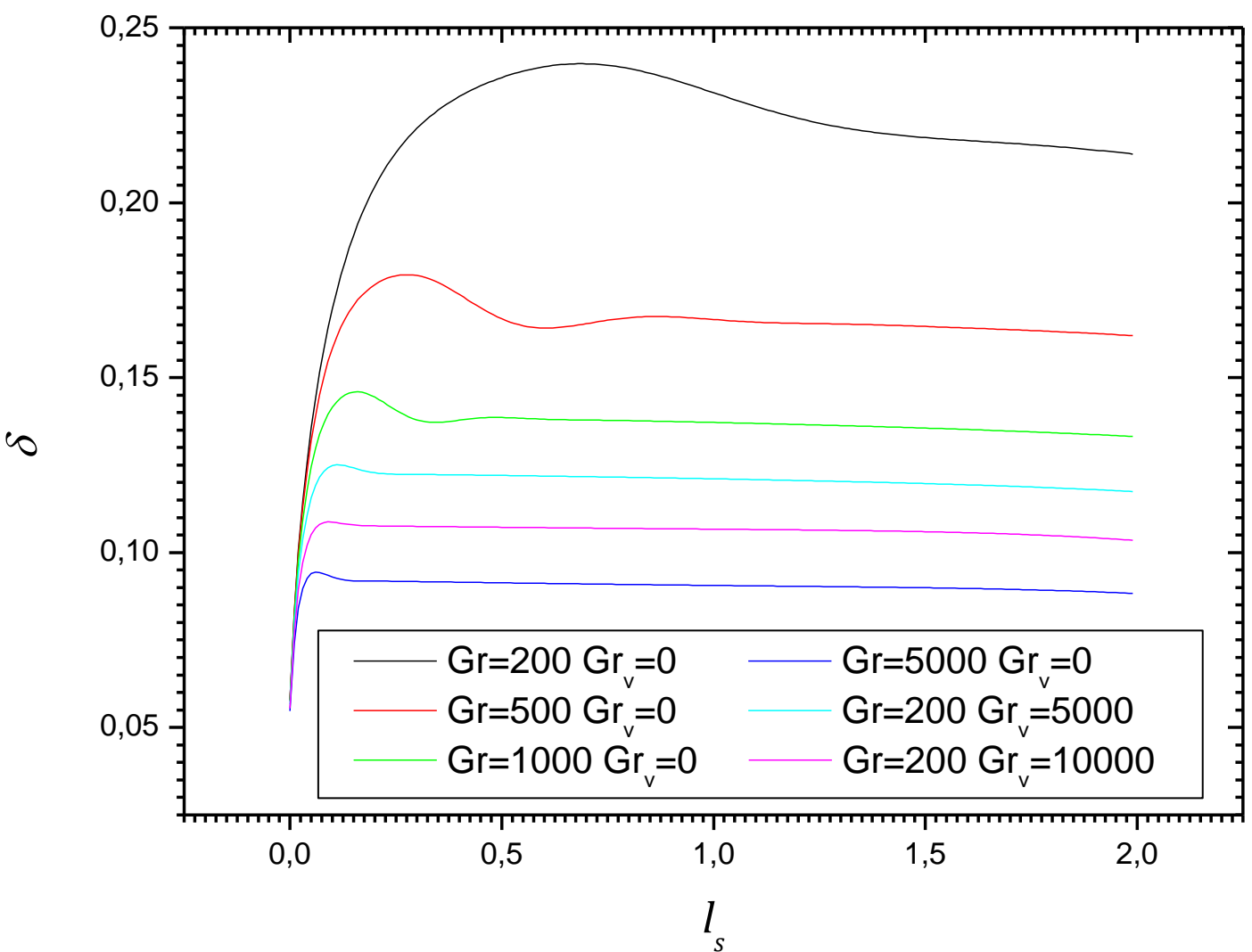

(b) 
Fig. 7. Axial segregation $C_{s, a v}$ of phosphorus in the silicon ingot (a) and solute boundary layer thickness $\delta$ at the interface in the melt (b) given as a function of the solidified length $l_{s}$ for $\operatorname{Pr}=0.0113, \mathrm{Sc}=12.74, k=0.332$, and different values of the Grashof number $\mathrm{Gr}$ and the vibrational Grashof number $\mathrm{Gr}_{\mathrm{v}}\left(\alpha=45^{\circ}\right)$. In (a), comparisons with respect to the modified Scheil-Gulliver model are given as dashed lines.

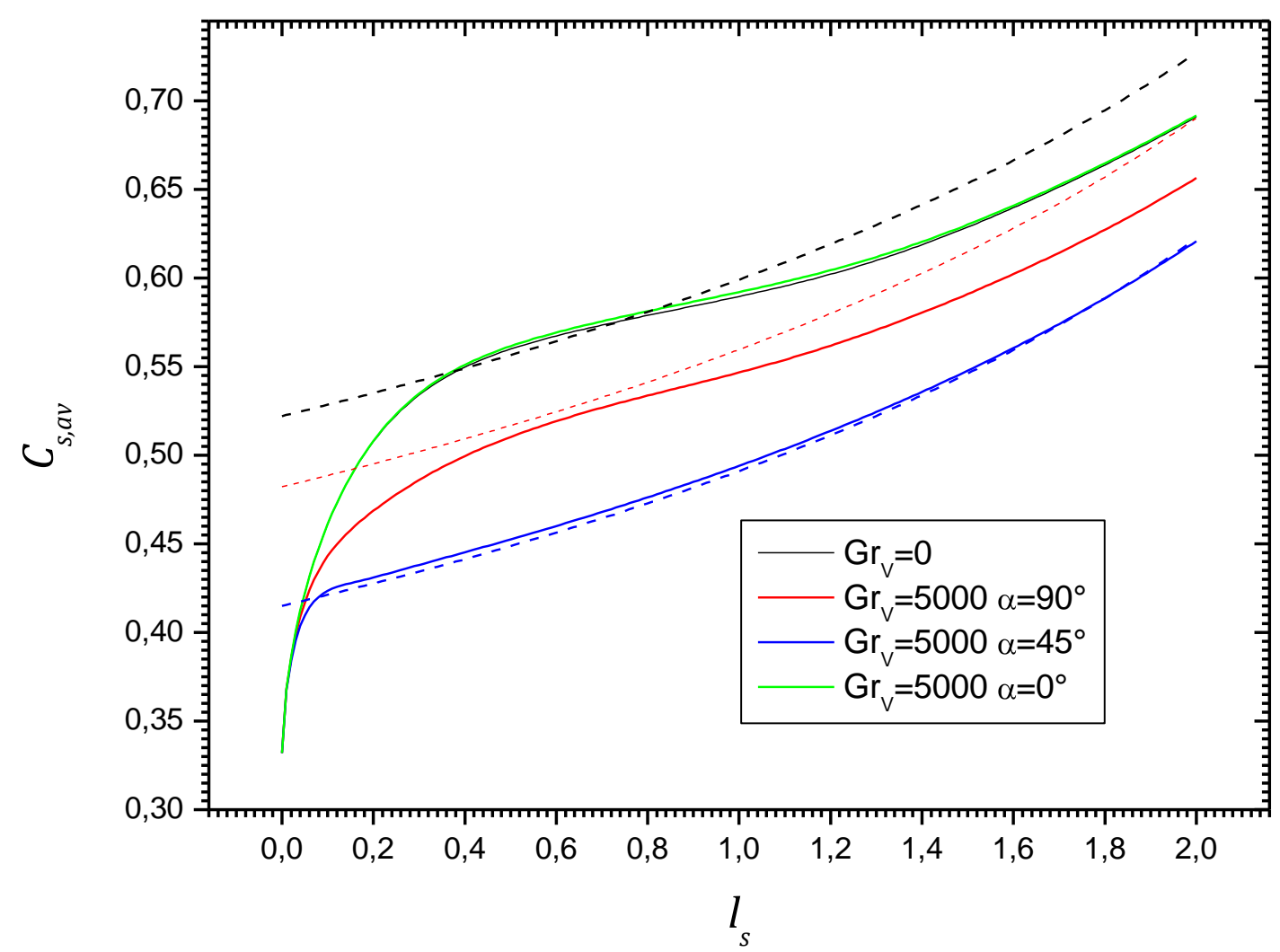

Fig. 8. Effect of the vibration direction on the axial segregation $C_{s, a v}$ of phosphorus in the silicon ingot $(\mathrm{Pr}=0.0113, \mathrm{Sc}=12.74, k=0.332, \mathrm{Gr}=200)$. The curves of $C_{s, a v}$ are given as a function of the solidified length $l_{s}$ for $\mathrm{Gr}_{\mathrm{v}}=5000$ and $\alpha=0,45$, and $90^{\circ}$, and for $\mathrm{Gr}_{\mathrm{v}}=0$ (without vibration). Comparisons with respect to the modified Scheil-Gulliver model are given as dashed lines. 


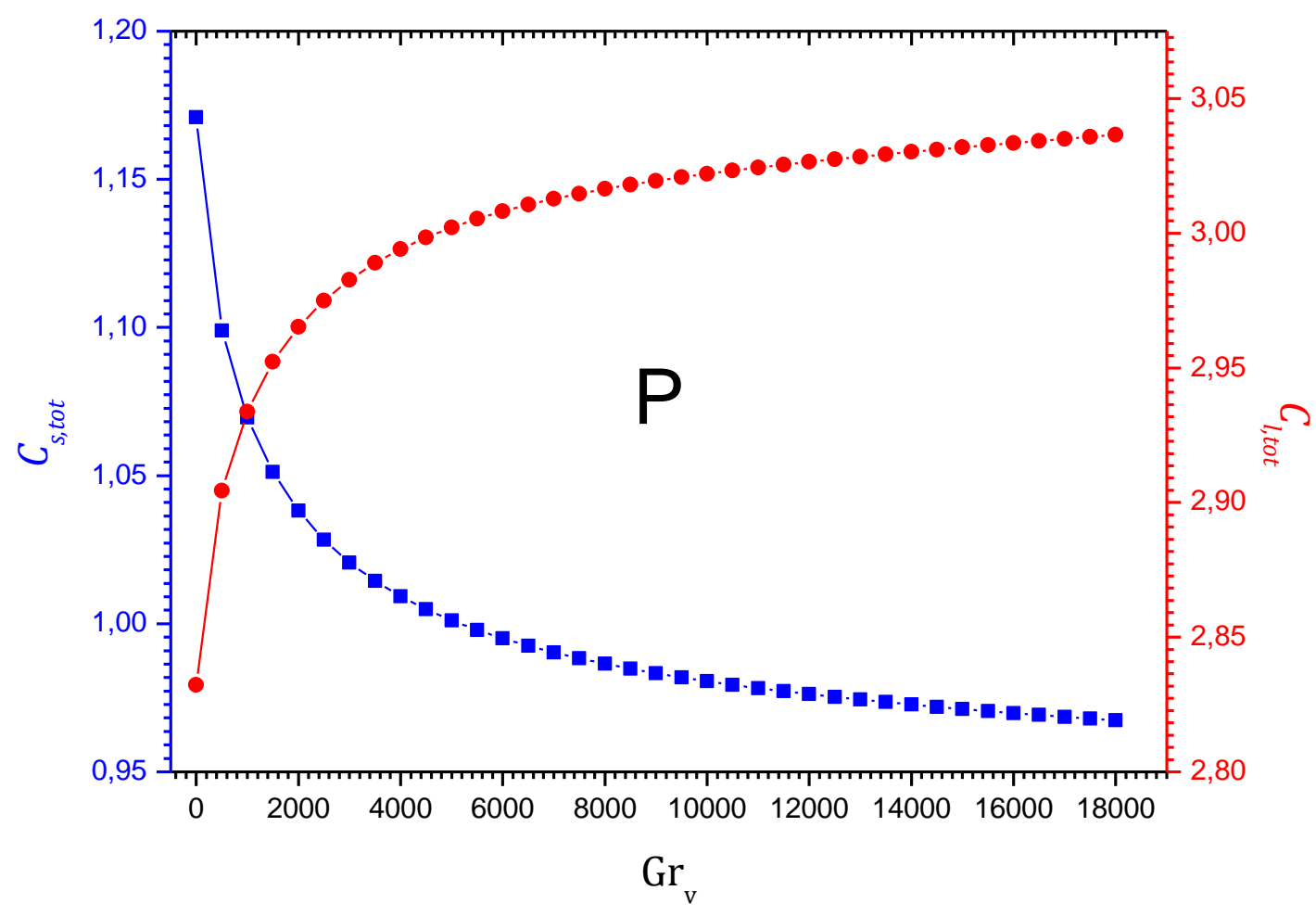

(a)

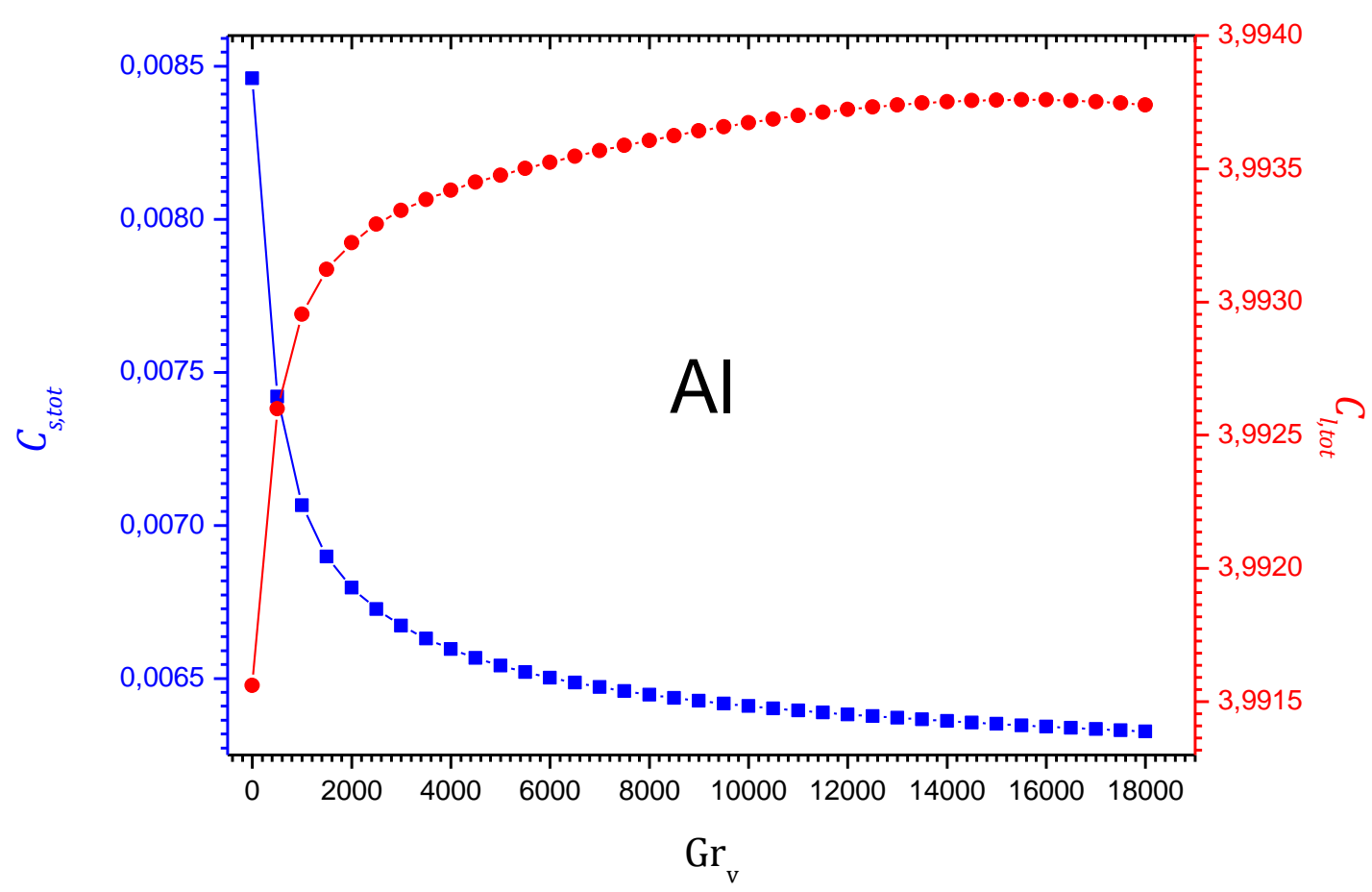

(b)

Fig. 9. Global impurity level in the silicon crystal $\left(C_{s, t o t}\right)$ and in the melt $\left(C_{l, t o t}\right)$ versus $\mathrm{Gr}_{\mathrm{v}}$ for the optimal vibration direction $\left(\alpha=45^{\circ}\right)$. The results are given for phosphorus (a) and aluminum (b) when half of the molten silicon has been solidified $(S=2, \operatorname{Pr}=0.0113, \mathrm{Gr}=$ 200). 


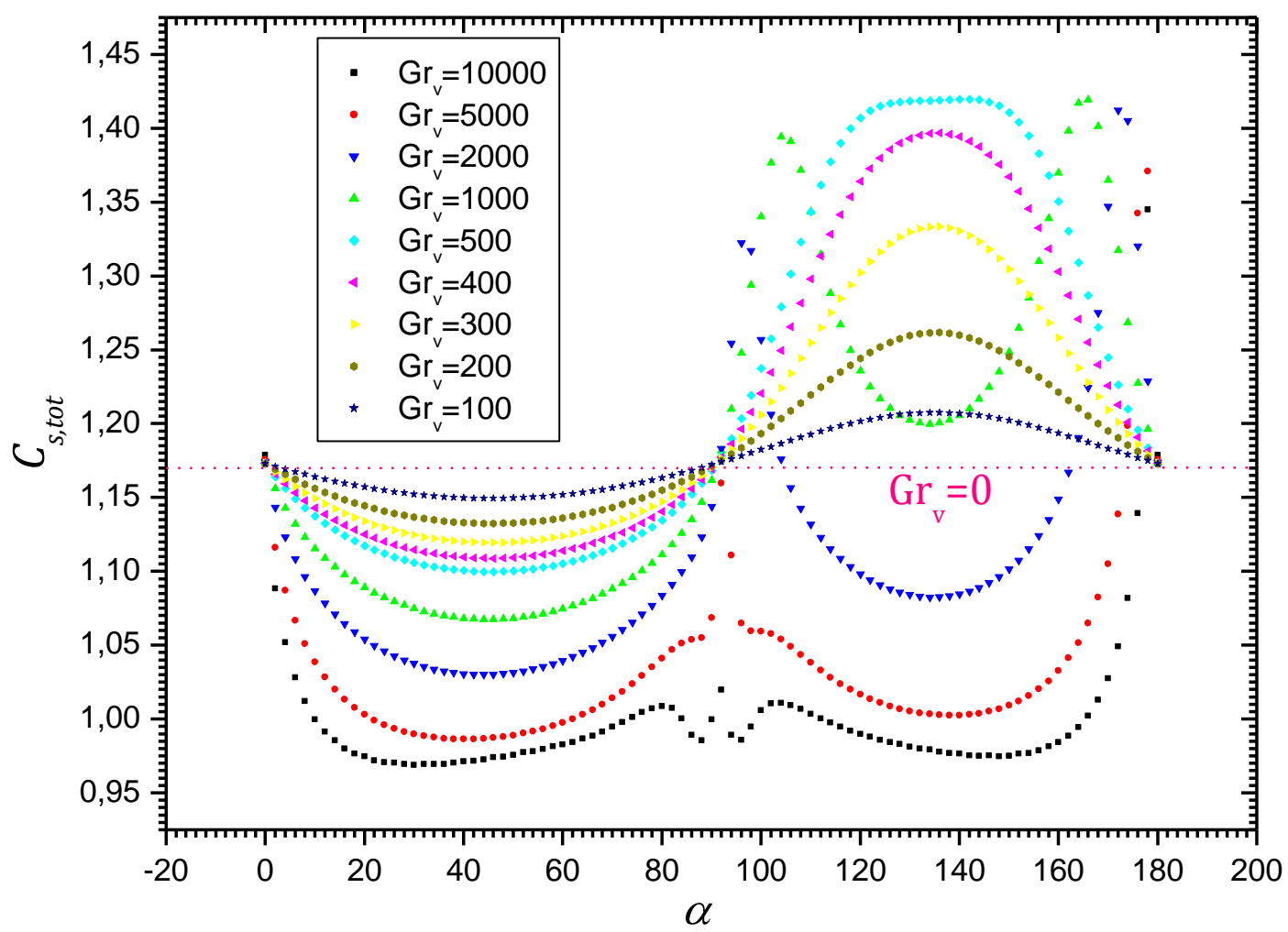

Fig. 10. Variation of the global impurity level for phosphorus in the silicon crystal, $C_{s, t o t}$, with the vibration angle $\alpha$ for $\mathrm{Gr}_{\mathrm{v}}$ values in the range 100-10000 $(S=2, \operatorname{Pr}=0.0113, \mathrm{Gr}=$ $200)$. The value of $C_{s, t o t}$ obtained without vibrations $\left(\mathrm{Gr}_{\mathrm{v}}=0\right)$ is given as a pink dashed line for comparison: the parts of the curves below this line indicate a purification due to the applied vibrations. 


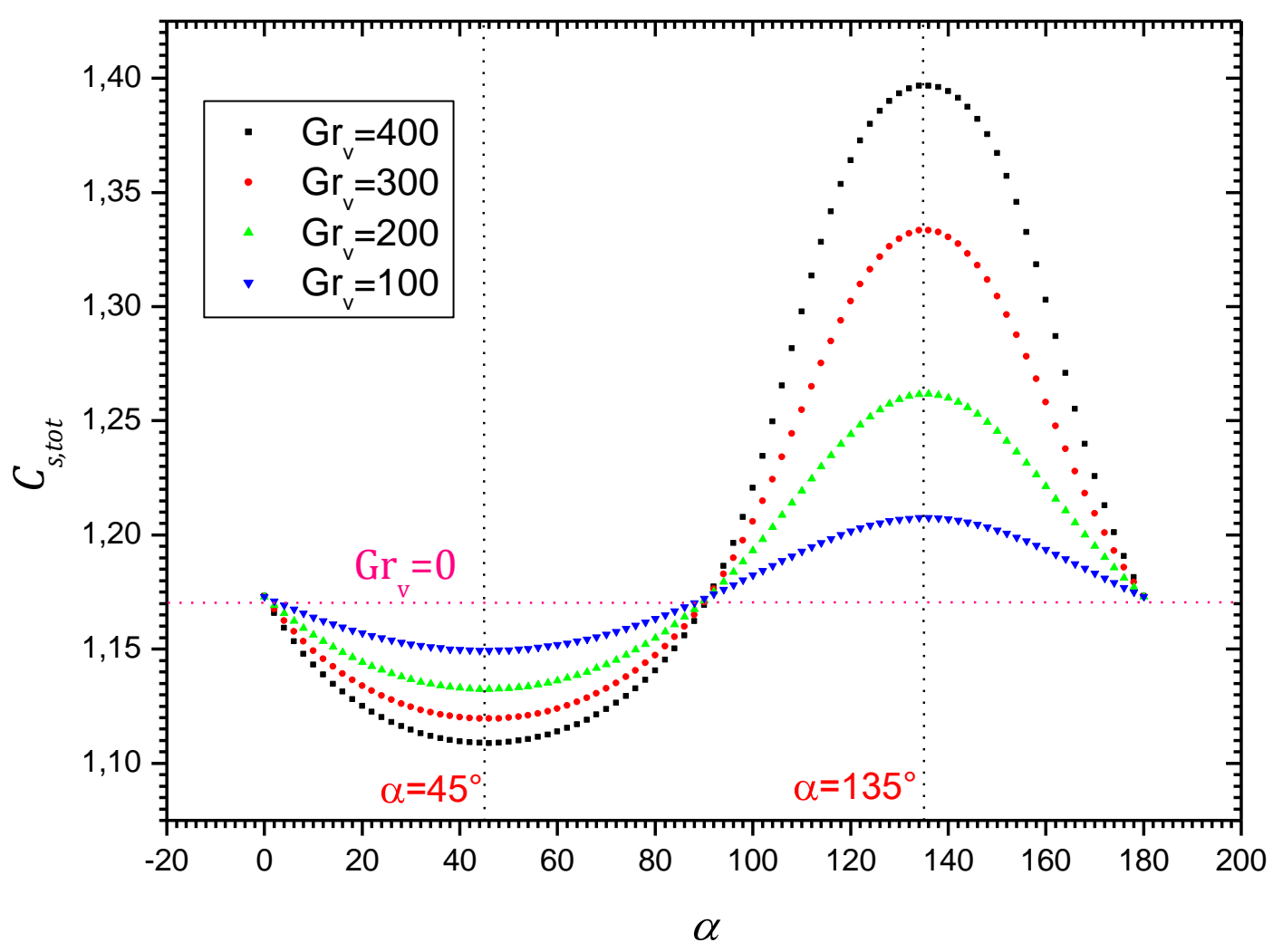

(a)

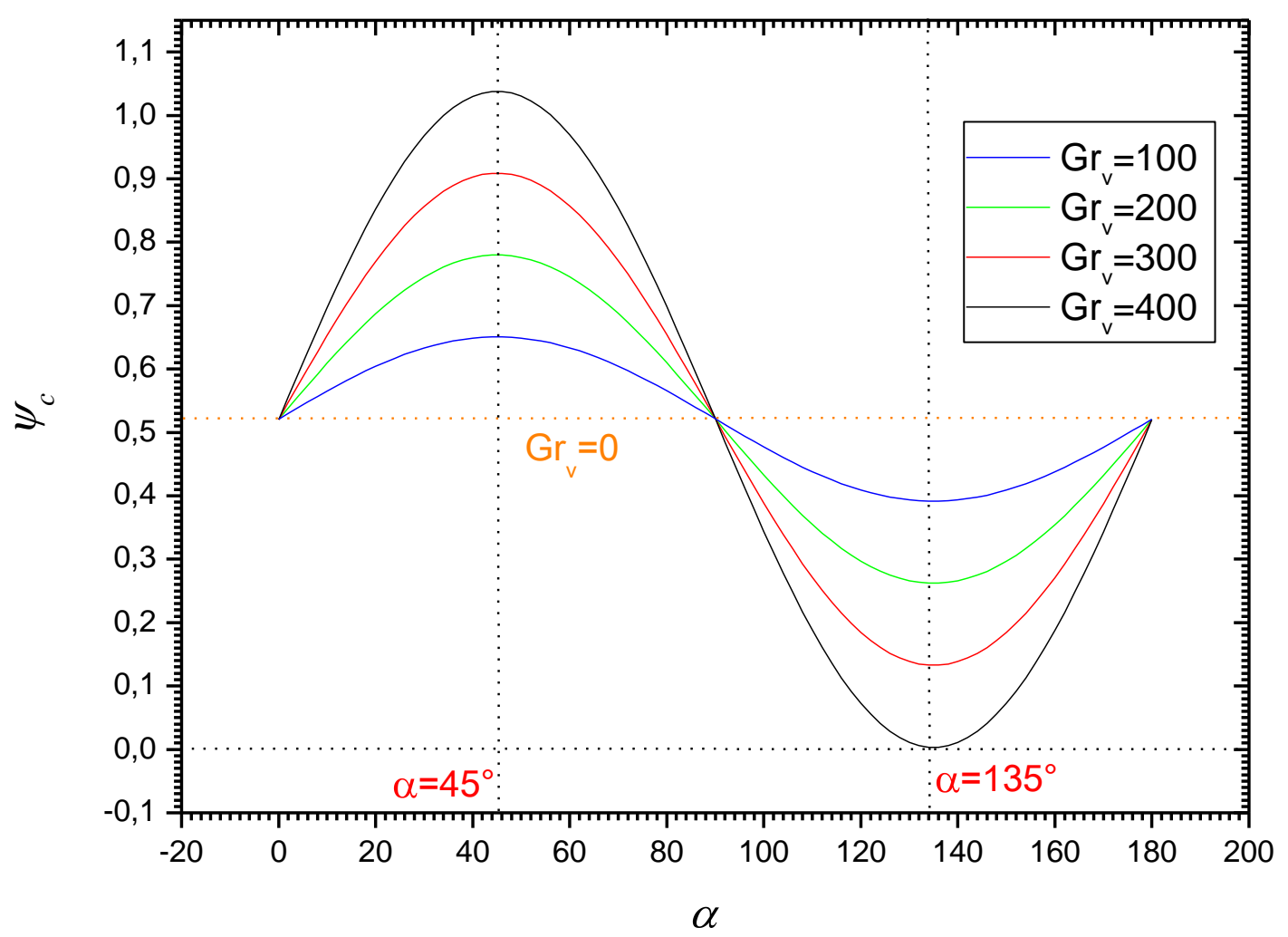

(b)

Fig. 11. Global impurity level for phosphorus in the silicon crystal $C_{s, t o t}$ (a) and stream function at the center of the melt zone $\psi_{c}$ (b) as a function of the vibration angle $\alpha$ for small 
$\mathrm{Gr}_{\mathrm{v}}$ values in the range 100-400 where flow inversion does not occur $(S=2, \operatorname{Pr}=0.0113$, $\mathrm{Gr}=200)$. The values of $C_{s, t o t}$ and $\psi_{c}$ obtained without vibrations $\left(\mathrm{Gr}_{\mathrm{v}}=0\right)$ are given as pink and orange dashed lines, respectively, for comparison.

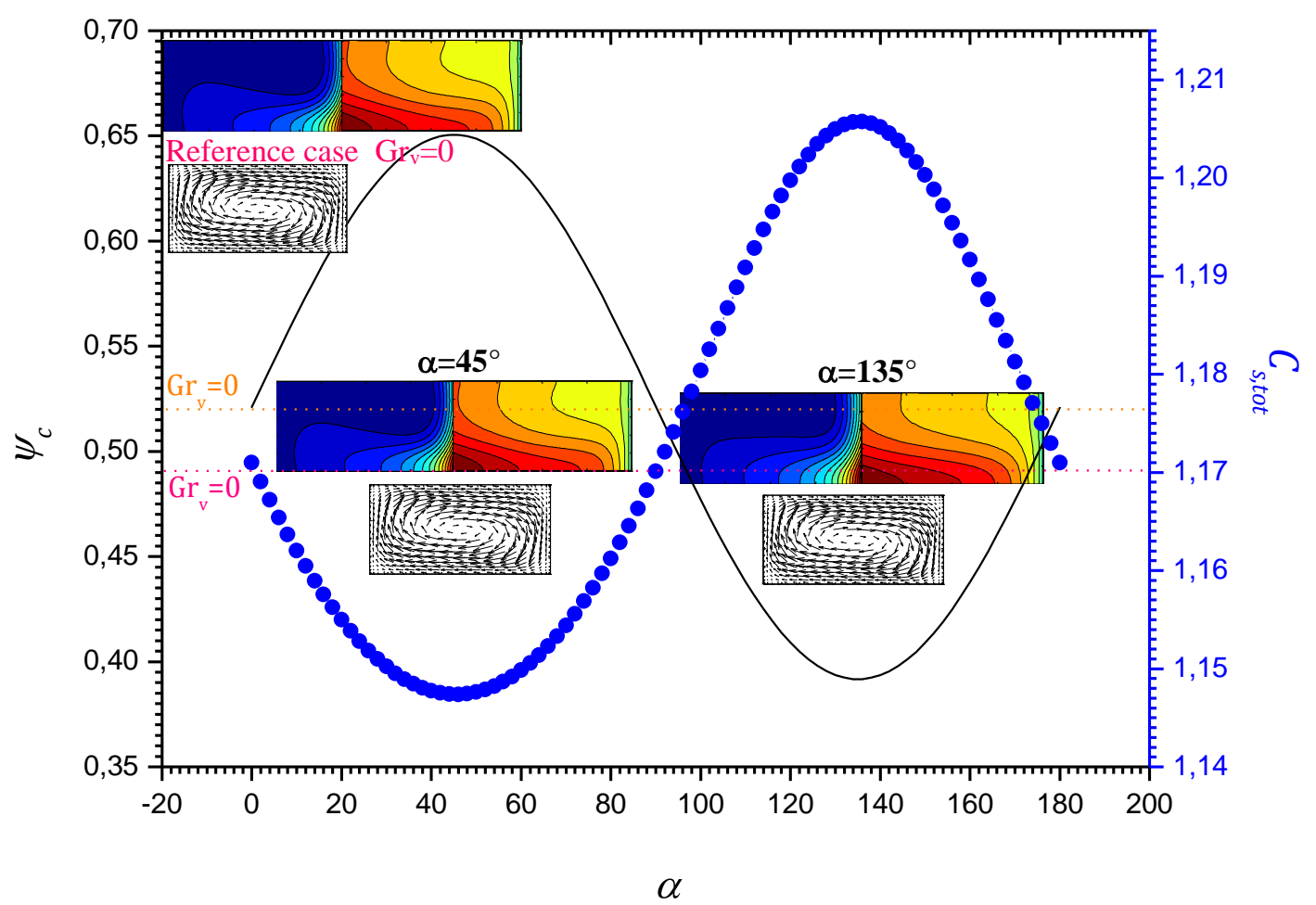

(a) 


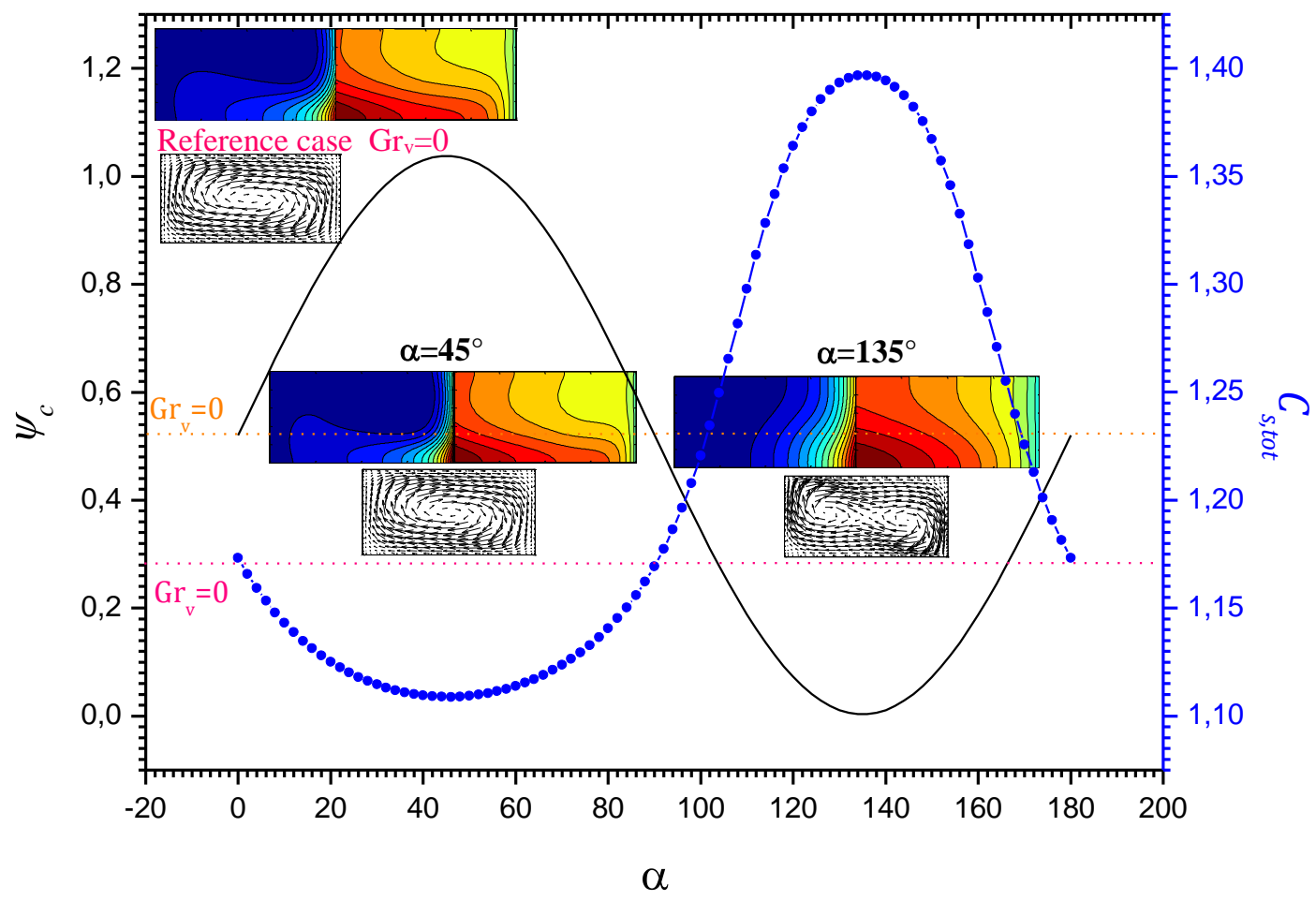

(b)

Fig. 12. Global impurity level for phosphorus in the silicon crystal $C_{s, t o t}$ and stream function at the center of the melt zone $\psi_{c}$ as a function of the vibration angle $\alpha$ for $\mathrm{Gr}_{\mathrm{v}}=100$ (a) and $\mathrm{Gr}_{\mathrm{v}}=400(\mathrm{~b})(S=2, \operatorname{Pr}=0.0113, \mathrm{Gr}=200)$. The values of $C_{s, t o t}$ and $\psi_{c}$ obtained without vibrations $\left(\mathrm{Gr}_{\mathrm{v}}=0\right)$ are given as pink and orange dashed lines, respectively, for comparison. Insets give velocity vectors and iso-concentration lines in the melt (left part) and in the crystal (right part) at $S=2$ for the vibration angles giving maximal variations $\left(\alpha=45^{\circ}\right.$ and $\left.135^{\circ}\right)$. The reference case $\mathrm{Gr}_{\mathrm{v}}=0$ is also given. 


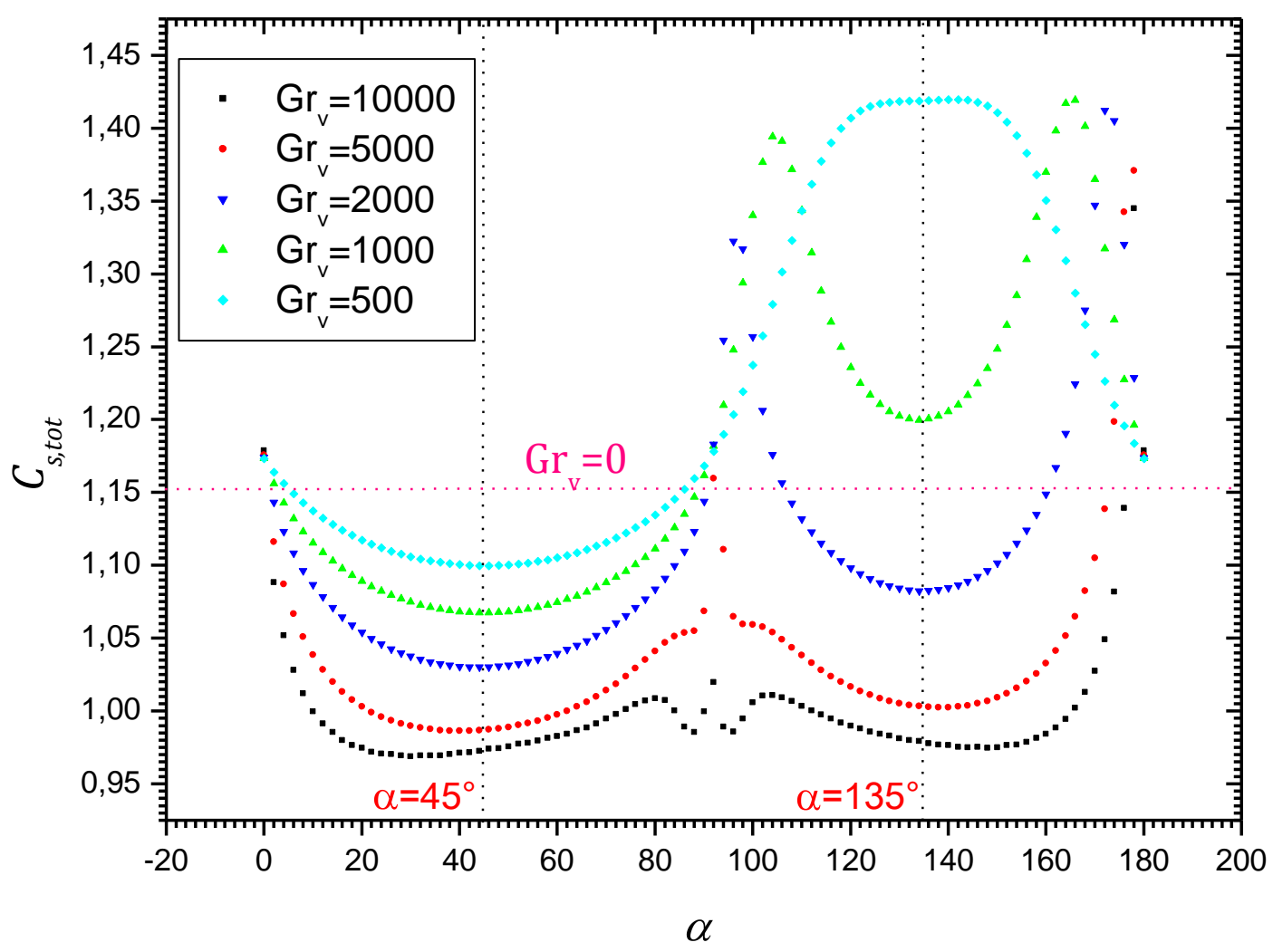

Fig. 13. Variation of the global impurity level for phosphorus in the silicon crystal $\left(C_{s, t o t}\right)$ with the vibration angle $\alpha$ for large $\mathrm{Gr}_{\mathrm{v}}$ values in the range 500-10000 where flow inversion occurs $(S=2, \operatorname{Pr}=0.0113, \mathrm{Gr}=200)$. The value of $C_{s, t o t}$ obtained without vibrations $\left(\mathrm{Gr}_{\mathrm{v}}=0\right)$ is given as a pink dashed line for comparison. The parts of the curves below this pink line indicate a purification due to the applied vibrations. 


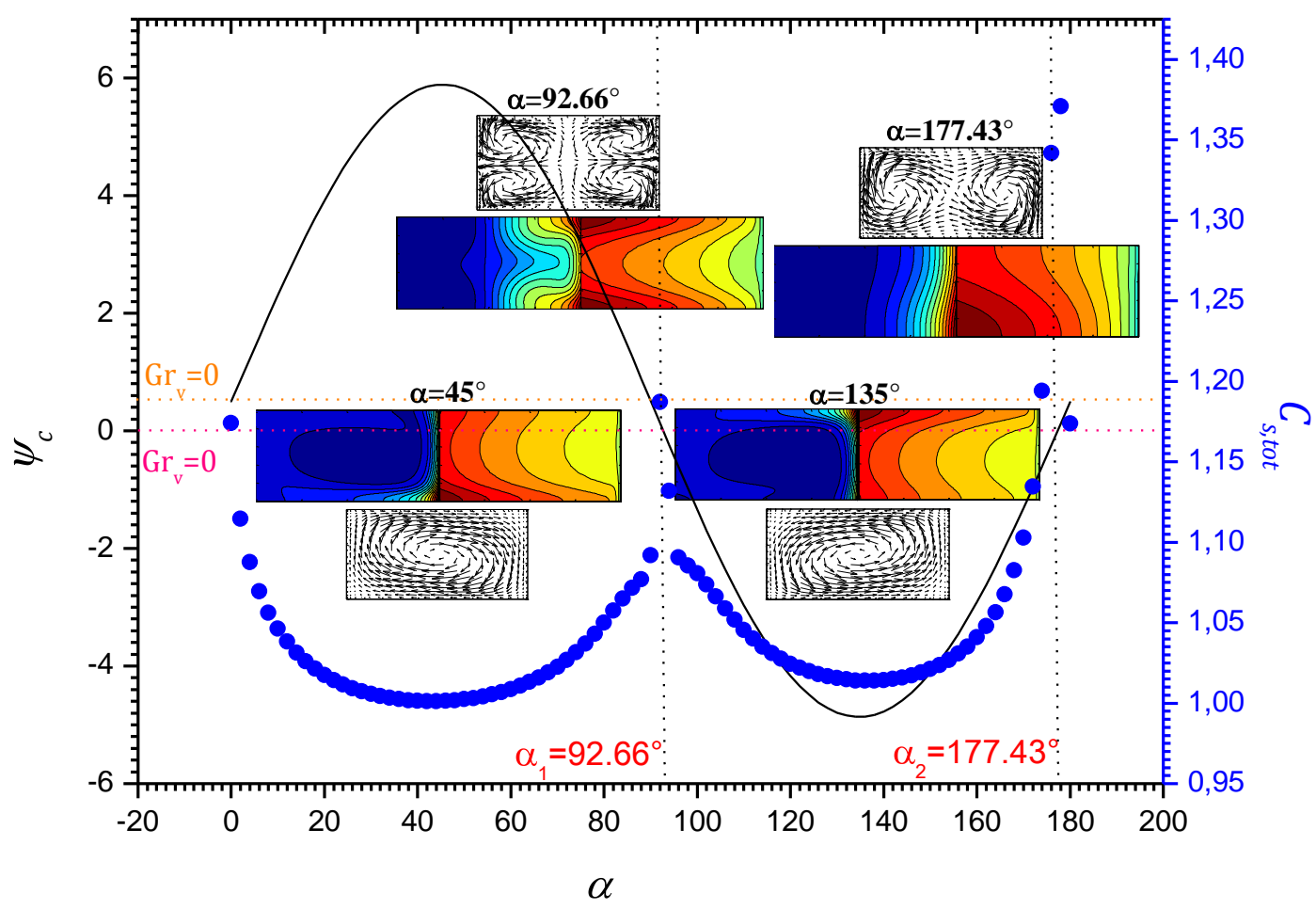

Fig. 14. Global impurity level for phosphorus in the silicon crystal $C_{s, t o t}$ and stream function at the center of the melt zone $\psi_{c}$ as a function of the vibration angle $\alpha$ for $\mathrm{Gr}_{\mathrm{v}}=5000(S=$ $2, \operatorname{Pr}=0.0113, \mathrm{Gr}=200)$. The values of $C_{s, t o t}$ and $\psi_{c}$ obtained without vibrations $\left(\mathrm{Gr}_{\mathrm{v}}=\right.$ $0)$ are given as pink and orange dashed lines, respectively, for comparison. Insets give velocity vectors and iso-concentration lines in the melt (left part) and in the crystal (right part) at $S=2$ for the vibration angles giving maximal purification $\left(\alpha=45^{\circ}\right.$ and $\left.135^{\circ}\right)$ and those at the limit of flow inversion $\left(\alpha_{1}\right.$ and $\left.\alpha_{2}\right)$. 


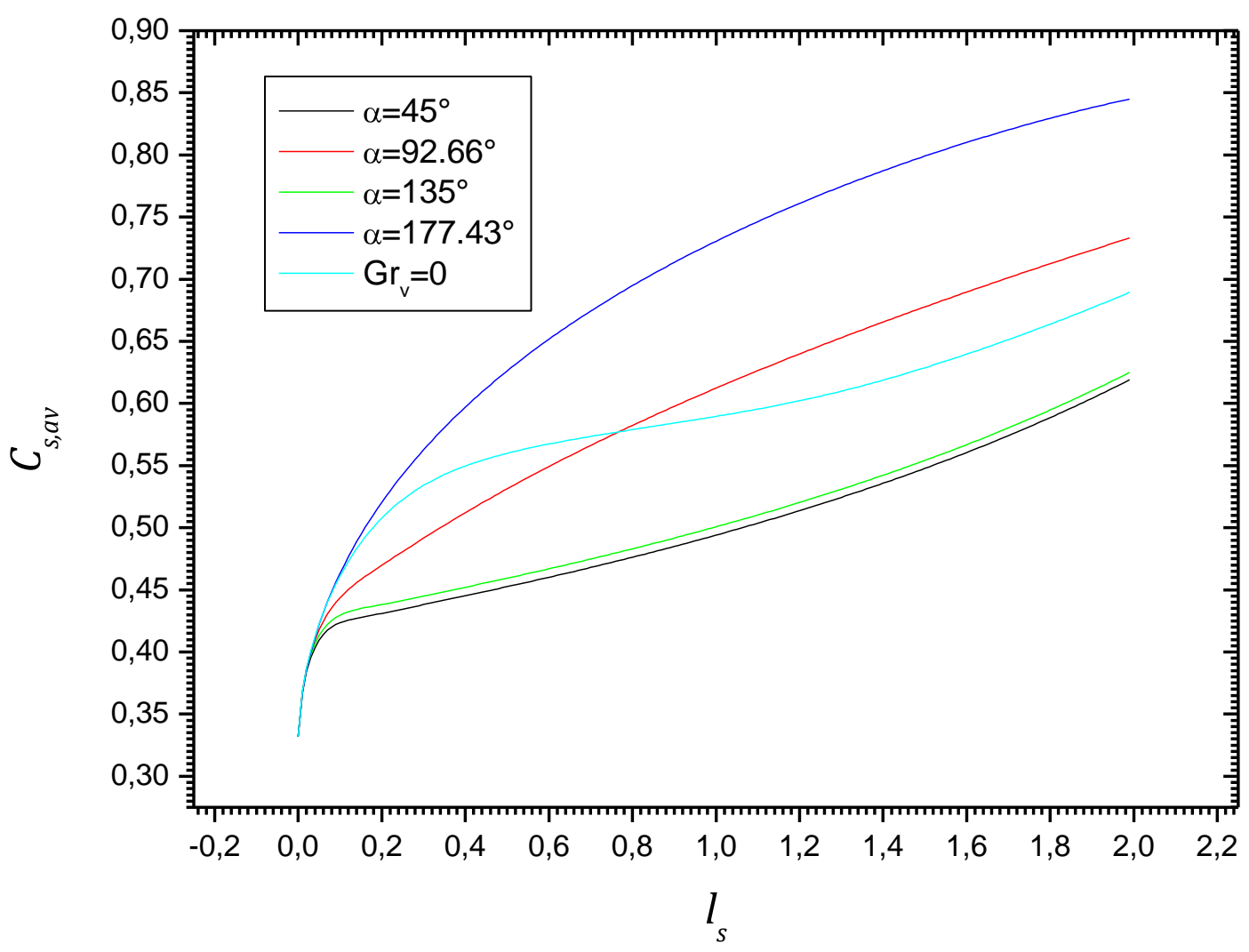

Fig. 15. Axial segregation $C_{s, a v}$ of phosphorus in the silicon ingot versus the solidified length $l_{s}$ for $\mathrm{Gr}_{\mathrm{v}}=5000$ and different vibration angles $\alpha(S=2, \operatorname{Pr}=0.0113, \mathrm{Gr}=200)$. The case without vibration $\left(\mathrm{Gr}_{\mathrm{v}}=0\right)$ is given for comparison. 